Research Article

\title{
Comprehensive Evaluation Model of Bearing Transportation Protection Effect of Bogie Traction Motor under Data Imbalance
}

\author{
Yi Liu, ${ }^{1,2,3}$ Qi Chang, ${ }^{1}$ Jiaxin Luo, ${ }^{1}$ LinLi, ${ }^{3}$ Junfeng Man, ${ }^{1}$ FenWei, ${ }^{4}$ Qinlin Chen, ${ }^{4}$ \\ and Yiping Shen $\left(^{4}{ }^{4}\right.$ \\ ${ }^{1}$ School of Computer Science, Hunan University of Technology, Zhuzhou, 412000 Hunan, China \\ ${ }^{2}$ CRRC Zhuzhou Electric Locomotive Co., LTD., Zhuzhou, 412000 Hunan, China \\ ${ }^{3}$ National Innovation Center of Advanced Rail Transit Equipment, Zhuzhou, 412000 Hunan, China \\ ${ }^{4}$ Hunan Key Laboratory of Mechanical Equipment Health Maintenance, Hunan University of Science and Technology, Xiangtan, \\ 411201 Hunan, China
}

Correspondence should be addressed to Yiping Shen; yiping1011@163.com

Received 3 October 2021; Accepted 5 November 2021; Published 6 December 2021

Academic Editor: Haidong Shao

Copyright (c) 2021 Yi Liu et al. This is an open access article distributed under the Creative Commons Attribution License, which permits unrestricted use, distribution, and reproduction in any medium, provided the original work is properly cited.

Under different transportation protection, the sample data of bogie traction motor bearings of urban rail vehicles are seriously unbalanced, and the fault diagnosis ability and generalization effect are poor, which makes it difficult to evaluate the protection effect of bearings effectively. In this paper, a multimeasure hybrid evaluation model based on compressed sensing is proposed to evaluate the effect of bearing transportation protection under data imbalance. Firstly, bearing vibration signals under different transport protection conditions were compressed and sampled, and the original high-Witt collection in time domain, frequency domain, and time-frequency domain was extracted. Then, a multimeasure mixed feature evaluation model of correlation, distance, and signal was constructed, and the optimal multimeasure combination strategy was optimized by using comprehensive sensitivity score evaluation index. Finally, an evaluation model of bearing protection effect based on unified feature index was constructed by using the best feature subset evaluated, and the unified indicator was quantified to characterize the protection effect of different protection states. The experimental results show that the model can effectively evaluate bearings under different transport protection.

\section{Introduction}

As an important part of the walking part of urban rail transit vehicles, the bogie plays the role of motion orientation, bearing, and vibration reduction and is also the ultimate executor of traction and braking and plays an important role in the safe driving of the train [1]. At the present stage, the bogies put into use in China are mainly based on welding. Due to the characteristics of the welding process itself, the weld position of the bogies is easy to transmit residual stress and deformation will have influence, and the traction and driving device of the vehicle will be directly related to the quality of force transmission, and whether the structure is safety and reliable will directly affect the operation safety of the train [2]. Traction motor is the key part of urban rail vehicle driving device, its operation state will directly affect the train performance and transportation efficiency, including rolling bearing which is one of the most widely used parts of traction motor, and the relevant research results show that the most prone to failure parts of traction motor is rolling bearing; bearing damage accounts for about $44 \%$ of traction motor failure [3]. Therefore, ensuring the quality of the bogie traction motor bearing is one of the important factors to ensure the safe, stable, and comfortable operation of the urban rail vehicles. Urban rail vehicles are often transported to the owner's site [4]. If the bearings are not protected during transportation, pseudocloth marks will occur due to the road turbulence, abnormal bearing sound during 
site operation, and eventually mass replacement, which seriously affects the manufacturer's product quality of the bearing and causes serious hidden dangers to the safe operation of urban rail trains.

The protection effect assessment of the traction motor bearing of urban rail vehicles under different transportation protection has two main difficulties:

(1) In the signal collection of the urban rail vehicle steering gear traction motor bearing, there is a serious data imbalance phenomenon. The main reasons of this phenomenon are as follows. (a) With the vehicle running speed and the complex environment, the data flow accelerates, and the amount of state data increases. Due to the development and deterioration of the equipment state, the shortening of the monitoring period will also lead to the increase of the state data volume. (b) The bearing produces vibration signals containing impact attenuation components under different transportation protection, all of which have obvious nonlinear behavior, which eventually leads to the spectral bandwidth of the signal, excessive number of data collection, and massive data bring great pressure on data transmission, storage, and processing. (c) Under the actual working condition, the number of bearing fault signal samples is generally far less than the normal state signal. In conclusion, the mentioned factors will cause unbalanced bearing dataset

(2) The fault diagnosis ability of the bearing is poor, and the protective effect assessment is difficult to be effectively evaluated. The reasons of this phenomenon are as follows: the complex rotating mechanical structure and bad operating environment make the fault mode usually show strong complexity [5], and the "underlearning" problem of fault feature sensitivity in the widely used single measure evaluation model and the common feature parameter extraction of different analysis fields can only state the information of mechanical equipment characterized by vibration signal be described from different angles. The above situation causes the failure to obtain the status information in the bearing fault diagnosis, so as not to provide an accurate data basis for the pattern recognition and then affect the subsequent classification performance and effect assessment

\section{Related Research Work}

The application object of previous research is generally limited to the error data with similar distribution under constant and stable conditions. Unfortunately, this restriction has little effect in real-life scenarios. The working conditions are complex and changeable, which makes it difficult to extract effective fault diagnosis representation in practical application by previous deep representation learning methods [6, 7]. To solve the serious data imbalance of bearing vibration signal under continuous high sampling, this paper adopts compression sensing (compressive sensing (CS)) theory also called compression sampling theory. A compressed sensing theory proposed by Donoho et al. [8-10], whose signal compression theory breaks through the Nyquist limit, can achieve less measurement compression sampling and complete high probability accurate reconstruction. Although the observation data is reduced, it contains enough original signal information for signal recovery to realize the "compression sampling" of the signal. The concept of this theory can be described as follows: for a collected time-domain target signal, as long as the signal is compression on a certain sparse transformation basis, a linear measurement matrix unrelated to the sparse transformation base and select an appropriate reconstruction algorithm to accurately reconstruct the signal based on the low-dimensional compression measurement. Currently, compressed sensing hotspots focus on three key problems: sparse representation, signal observation, and signal reconstruction $[11,12]$. Early studies performed limited sampling studies using the compressibility of signals and sampled continuous signals using a fixed structural basis function at twice the information rate rather than twice the sampling frequency [13]. The notion of the uncertainty principles of sparse representation is first proposed by Donoho et al. [14]. Based on this result, El Ad and Bruckstein [15] further discuss the uniqueness of the sparse representation and prove the boundary conditions for the exact sparse reconstruction. Tropp [16] proposed more general conditions, unifying the reconstruction conditions for the sparse representation problem of the $l_{0}$ and $l_{1}$ norm constraints. Candes et al. [16] propose the exact reconstruction principle that further demonstrates the uniqueness of the sparse representation problem and discuss the stability, robustness, and the extension of the algorithm. Baraniuk et al. $[17,18]$ proposed that constrained isometries provide a theoretical basis for the observation matrix design and signal reconstruction. In recent years, CS has made some progress in image compression, face recognition, radar imaging, communication, and other fields [19], but at present, the research in the field of mechanical equipment fault diagnosis is still widely involved, and little research is applied in the field of urban rail vehicles.

The fault feature sensitivity "underlearning" problem in the single measure evaluation model and the feature parameter characterization information extracted by different single analysis domain often have significant undercompleteness. It is not difficult to find that extracting the multidomain multifeature parameter is a necessary guarantee to comprehensively describe the equipment fault state mode. Meanwhile, the feature weighted has rich feature sensitivity information to the original feature set with eigenweights without losing any feature parameter, giving the weighted feature set with better category discrimination ability. According to the principle of feature weighting, the calculation of its weight coefficient is closely related to various types of feature evaluation criteria, from which various characteristic weighting methods based on typical characteristic evaluation measures or their improved versions are born, for example, fault feature weighting based on compensation distance evaluation technology [20], nuclear space distance 
measure [21], feature weighted scheme based on entropy, mean variance and mutual information, feature weighted based on joint Laplacian score, and feature weighting based on Fisher linear discrimination analysis. Moreover, Sáez et al. [22] proposed a novel feature weighted scheme based on interpolation method and Kolmogorov-Smirnov parametric statistical test for the performance improvement limited to noise-containing, redundancy, and weak correlation features; Ismail and Frigui [23] proposed a robust unsupervised learning algorithm for finite generalized Dirichlet hybrid model for realizing fuzzy clustering and feature weighted of noise-containing high-dimensional data. Similar to the selection of the optimal feature subset in feature selection, the determination of feature-weighted weight coefficient can be treated as a class of combinatorial optimization problems, so various evolutionary algorithms (including taboo search, harmonic search, and multiobjective optimization algorithm) [24] have been introduced into the construction of feature-weighted framework. However, most of the existing weight coefficient calculation methods based on feature evaluation criterion learn the sensitivity of features from a single measure such as distance, information, and correlation, which often causes poor learning results of fault feature sensitivity, which is not conducive to the improvement of feature clustering and classification performance. Based on the research of scholars, this paper proposes a multimeasure mixed evaluation model for accurate and comprehensive evaluation of bearings.

This paper studies the bogie traction motor bearing in urban rail vehicles and evaluates the reasonable bearing transportation protective measures during road transportation. A multimeasure hybrid evaluation model based on compression perception is proposed and applied to the transportation protection example of Ningbo Line 3 bearings, to verify the feasibility and technical advantages of the proposed method, evaluate the best protection measures to reduce the failure rate of bearings, provide theoretical basis and technical reference for the characterization of the transportation protection effect of urban rail vehicles, and then provide certain guarantee for the safe operation of urban rail vehicles. The overall technical route for evaluating the protection effect of urban rail vehicle bogie traction motor bearings under different transportation protection is shown in Figure 1.

\section{Compression Sampling of Bearing Vibration Signal in the Field of Rail Transit}

The status data of the mechanical equipment can reflect the real-time operation state of the equipment, which is an important basis for obtaining the changing trend of the equipment status and analyzing the root cause of the equipment failure. In the fault diagnosis of rail transit field, status data is an indispensable and important resource related to the operation, monitoring, and management of urban rail vehicles. In order to accurately grasp the health state of the equipment, it is the urgent problem to solve the imbalance of bearing dataset. Compression sensing theory provides new ideas for signal acquisition, analysis, and feature extrac- tion, which is currently rarely involved in fault diagnosis in the field of rail transit. Compact data is used to obtain compressed data and deal with the storage of massive data, analyze compressed data from compressed data, reduce analysis and calculation links, save computational resources, and improve the efficiency of monitoring and diagnosis. The application of compression sensing theory to the signal sampling of equipment in rail vehicles provides an effective solution to the problem of data imbalance and its application in the storage, transmission, and analysis of urban rail vehicle equipment data. CS first performs the sparse transformation processing of the original signal and then measures the sparse data with the observation matrix, obtains the observation value far lower than the original signal dimension, and then restores the signal reconstruction algorithm of the compression perception theory framework to obtain the reconstruction signal with very little error with the original signal. The specific process is shown in Figure 2.

CS has been widely studied in many areas, but relatively little in the field of mechanical troubleshooting. This paper uses CS technology for the vibration signal of vehicle key components in the field of urban rail transit. In the whole evaluation process of the traction motor bearing of urban rail vehicle bogie, the processing process of compression sensing from the processing process of bearing vibration signal is mainly divided into two parts: one, acquisition and compression of signal; two, data reconstruction and analysis. The processing process of the bearing signal of the traction motor is shown in Figure 3.

3.1. Sparse Representation of the Signals in the Compressed Sampling. Sparse representation of signal in perception observation problem: compressed perception theory is the inverse problem of sparse representation theory, whose theory foundation implies the basis of signal sparsity. Shown in Figure 4 is a schematic diagram of sparse representation theory. The sparse representation of the signal is to transform the signal to a particular sparse space, yielding a small value of most of the transformation coefficients, namely, the resulting transformation coefficients are sparse or approximately sparse. Finding a base or compact frame $\Psi$ makes the signal $x$ sparse on $\Psi$ and finding the transform coefficients: $S=\Psi X$ where $S$ is an equivalent or approximated sparse representation of $X$. The choice of transform basis $\Psi$ can be some kind of basis that has been widely used, such as wavelet base, Fourier base, and local Fourier base. In addition, a compact framework can be used to make a sparse representation of signals such as curve waves and contour waves, two classes of transform bases with better directivity [25]. The prerequisite for compressed sensing is that the signal must be sparingly sparse. For most signals, it is usually not sparse, but it is always able to find the appropriate base for the sparse transformation of the signal.

3.2. Selection of the Observation Matrix in the Compressed Sampling. Main content of the signal observation research: how to design the $M \times N$-dimensional observation matrix $\Phi$, unrelated to the sparse basis to ensure that after the signal $x$ decreases from $N$ dimension length to $M$ dimension, the 


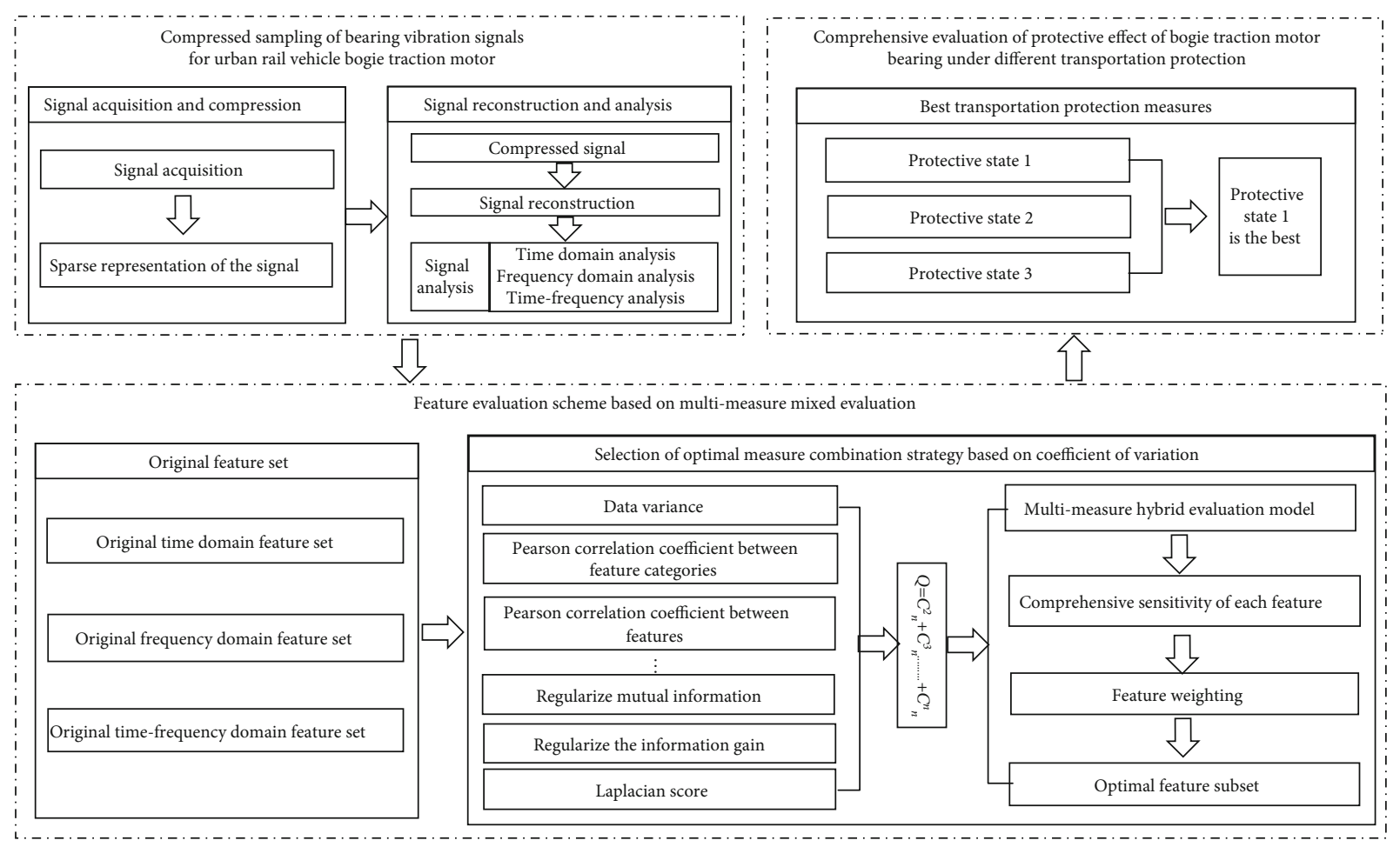

FIgURE 1: Technical schematic diagram of a multimeasure mixed evaluation model based on compression sensing.

\begin{tabular}{|c|c|c|c|}
\hline Sparse signal $\mathrm{x}$ & $\begin{array}{c}\text { Sparse transformation } \\
\theta_{i}=<\mathrm{X}, \varphi_{i}>=\varphi_{i}^{T} x\end{array}$ & $\begin{array}{l}\text { To reconstruct the signal } \\
\min \|\theta\|_{\text {os.t. }} y=A^{\operatorname{cs}} \theta\end{array}$ & $\begin{array}{c}\text { The observation matrix } \\
y=\Phi x\end{array}$ \\
\hline
\end{tabular}

Figure 2: Compression perception flowchart.

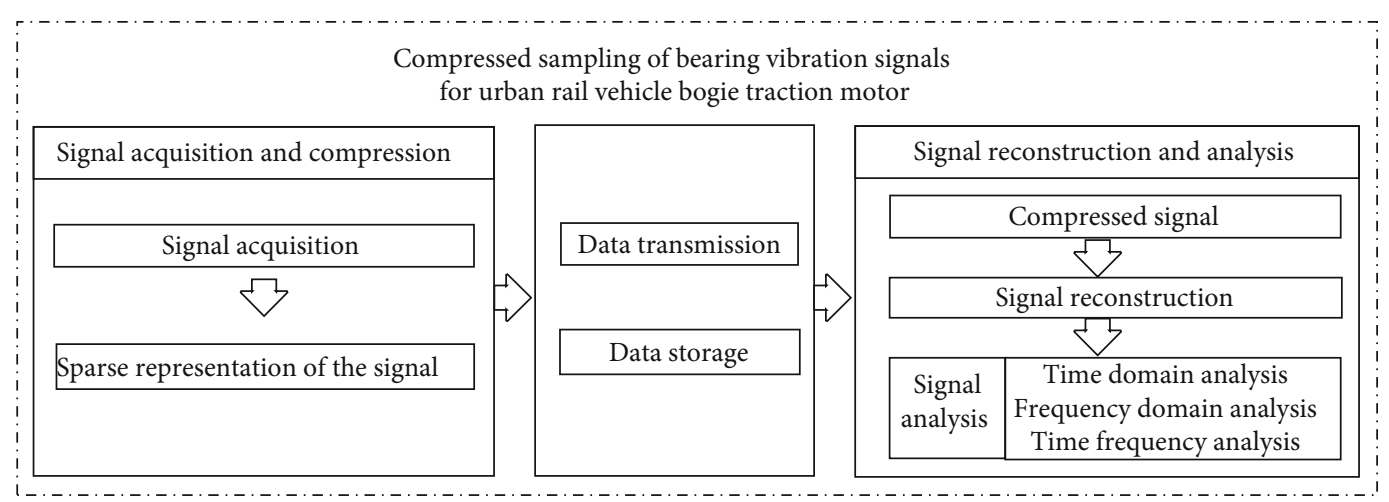

Figure 3: Signal processing process based on compression sensing for urban rail vehicle bearing.

main information is still not lost, and the original signal $x$ can be recovered from the dimension-dimensional signal $y$. The core of signal observation: to study sampling protocols that can effectively extract useful information in the original signal and to obtain as few sampling points as possible. The need $x$ to design a stable $m \times n$ dimensional observation matrix $\Phi$ is required to obtain the $S$ set $Y=\Phi S=\Phi \Psi^{T} X$. This process can also be represented as a signal $x$ for nonadaptive observations via matrix $A^{c s}: Y=A^{C S} X$ (where $A^{C S}$ $=\Phi \Psi^{T}$ is called the CS information operator). The concern is the selection of the observation matrix $\Phi$, which needs to guarantee that the important information is not broken when the sparse vector $S$ drops from $n$ dimension to $m$ dimension. In compressed sensing theory, an important criterion for judging whether a matrix can become a measurement matrix is limited isometric property (RIP). For example, for the $k$ sparse vector $S \in R^{N}$, when it satisfies formula (1), the measurement matrix $\Phi$ satisfies the RIP. Most random matrices satisfy RIP, such as Gaussian and Bernoulli stochastic measurements [17]. 


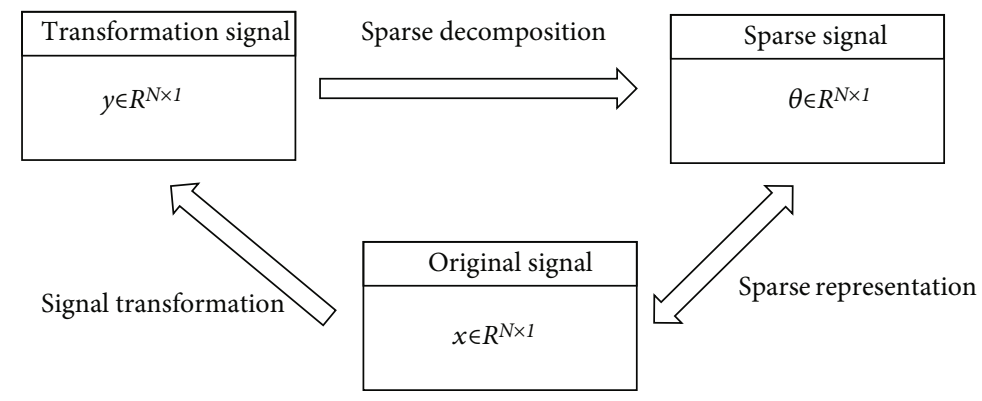

FIGURE 4: Sparse representation of theoretical diagram.

$$
(1-\varepsilon)\|S\|_{2} \leq\|\Phi S\| \leq(1+\varepsilon)\|S\|_{2}
$$

3.3. Design of the Reconstruction Algorithm in the Compression Sampling. The reconstructed recovery signal mainly solves the owed problem under a given sparse constraint and recovers the original signal with high probability from partially sampled data. The reconstruction of signals refers to the reconstruction of the original signal $y \in R^{M \times 1}$ from the dimension reduction signal $x \in R^{N \times 1}$. The key problem is how to design efficient, robust, and stable reconstruction algorithms. At the present stage, the CS reconstruction algorithm can be roughly divided into four categories: the first class: greedy iterative algorithm, aiming for the combinatorial optimization problem, which mainly take the link between signal and atomic dictionary as a more effective or nonzero way to measure atoms (coefficients). The fundamental principle is to find the support set of sparse vectors in an iterative way and to reconstruct the signal using a constrained support least squares estimation. The second class: convex optimization algorithm or optimization approximation methods, which find approximations of the signal by transforming nonconvex problems into convex problem solutions. The third kind of algorithm: based on the reconstruction algorithm proposed by the Bayesian framework, this algorithm takes into account the signal temporal correlation, especially when the signal has a strong temporal correlation, which can provide superior reconstruction accuracy than other reconstruction algorithms. Class 4: other algorithms: these methods support rapid signal sampling and reconstruction by packet testing, such as Fourier sampling, chain tracking, and Heavy Hitters on Steroids (HHS) tracking.

\section{Evaluation Model Based on Multimeasure Mixed Evaluation Based on Compression Sensing}

4.1. Compression Sensing of the Bearing Vibration Signal of the Bogie Traction Motor. The observation matrix dimension required to reconstruct the signal in the compressed sensing theory process is independent of the data sampling rate and the maximum frequency of the signal and is only directly related to the sparsity. To achieve compression sampling, the necessary sparsity processing of the signal is necessary to obtain the sparse signal in a specific space. In real life, the signal data is mostly nonsparse and requires some spatial transformation methods. In this paper, we analyze the sparse representation performance of bearings using three common discrete cosine transform (DCT) [17], discrete Fourier transform (DFT) [22], and discrete wavelet transform (DWT) [26]. In order to intuitively analyze the sparse performance from the perspective of the quantization indicators, $2 \%$ of the threshold $\varepsilon$, data $x$ peak value is first set as the threshold interval. That is, $\varepsilon=2 \%\left|\max \left(x_{i}-\min \left(x_{i}\right)\right)\right|$, assumes the data points between data values as $[-\varepsilon, \varepsilon]$ to 0 , and then exhausters the number of nonzero elements in $N_{0}$ data points in $x$, defines the sparse ratio $\eta_{2 \%}, \eta_{2 \%}=N_{0} / N$, where $N_{0}$ represents the number of data with numerical nonzero, the less sparse than $\eta_{2 \%}$, the better the sparse ability of the signal. The generalized orthogonal matching tracking algorithm (GOMP) is chosen as the reconstruction algorithm, enter the observation matrix $\Phi^{P \times l}$, probational measurements obtained from the observation matrix $y^{p \times l}$, sparsity degree $k$, select the number of atoms $S,(S \leq K \& S \leq p / K)$ the output is the reconstructed signal $\widehat{x}$. GOMP algorithm training procedure: first initialize the residual value of $r_{0}=$ $y$, index set $\Gamma_{0}=\varnothing$, number of changes $k=1$ (line 1); continuous search for $S$ matching atoms, $k=k+1$, search out the $S$ atoms with the largest residual product change its corresponding index value added, $P=P_{S}||\left\langle r_{k-1}, \Phi_{P S}\right\rangle\left|=\max _{M}\right|$ $\left\langle r_{k-1}, \Phi_{j}\right\rangle \|, i \in[1, S], \Gamma_{K}=\Gamma_{K-1} \cup P$ (lines 2-7); update the candidate support set as well as the residual and correlation coefficients. The corresponding support set $\Phi_{\Gamma_{K}}$ is obtained using the updated candidate atomic index set $\Gamma_{K}$. Calculate the updated residual and correlation coefficients, $\widehat{x}_{T_{K}}=\Phi_{\Gamma_{K}}^{+}$ $y, r_{k}=y-\Phi_{\Gamma_{K}} \widehat{x}_{\Gamma_{k}}$. If the iterative stop condition meets $k<$ $\min (K, p / S)$, the iterative process returns to step 2; otherwise, the iteration stops with the reconstruction signal $\widehat{x}=$ $x_{\Gamma_{k}}$, specifically, as shown in Algorithm 1.

4.2. Failure Characteristic Extraction of Bearing Vibration Signal of Bogie Traction Motor. With the failure of a part of the bogie or part of the bogie, the time domain waveform of the bogie state signal and the corresponding spectrum are different from the normal state, so it is considered that the characteristic parameters of the signal time domain and frequency domain distribution information are feasible to reveal the occurrence of the bogie failure. In addition, considering that the bearing is a rotating machine, the fault signal has low signal-to-noise ratio, nonlinear, and 


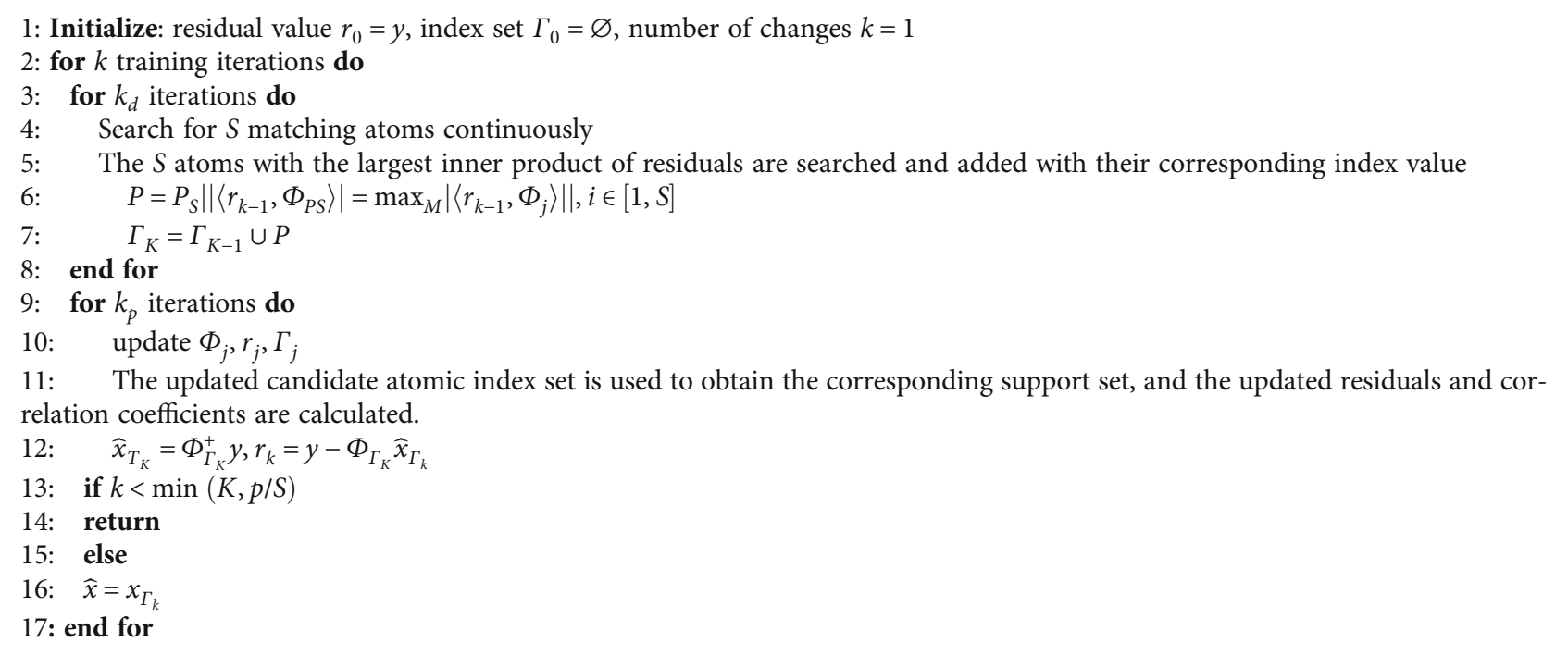

Algorithm 1: GOMP training process.

nonstationary characteristics, and the characteristics of the time-frequency analysis methods (such as short-frequency Fourier transform, wavelet transform, and EMD) have higher fault state information ability than the time domain and frequency domain characteristic parameters. In order to obtain the bearing fault state information as fully as possible, the experiment selects 10 time domain and 5 frequency domain and 10 time-frequency domain energy and LempelZiv frequency domain parameters. The selected time domain feature parameters form the original time domain feature set, $\left(T_{1}, T_{2}, \cdots, T_{10}\right)$ (respectively: peak, mean, variance, harmonic average, margin index, cliff index, wavedness index, pulse index, peak index, and deviation index). The frequency domain characteristic parameters form the original frequency domain characteristic sets $\left(F_{1}, F_{2}, \cdots, F_{5}\right)$ (respectively: mean frequency, center of gravity frequency, root mean square frequency, standard difference frequency, and cliff frequency). Time-frequency characteristic parameters are based on the adaptive decomposition of the original fault signal using EMD. Energy features $I M F=\left[\mathrm{IMF}_{1}, \mathrm{IMF}_{2}\right.$, $\left.\cdots, \mathrm{IMF}_{5}\right]$ extract the first five eigenmode components containing useful information and Lempel-Ziv complexity features $L=\left[L_{1}, L_{2}, \cdots, L_{5}\right]$.

\subsection{Comprehensive Characteristic Index of Bearing Vibration} Signal Based on Multimeasure Mixed Evaluation Model. The extraction of multicategory fault characteristic parameters is usually regarded as the basic guarantee for accurate fault diagnosis. However, if it is applied improperly, it is difficult to improve the fault diagnosis accuracy, because the sensitivity of the characteristic parameters is different. If the original Gavett collection is directly used as the input of the subsequent classifier, the advantage of sensitive characteristics on fault classification cannot be outstanding, and the correlation between nonsensitive characteristics will weaken the classification effect and improve the fault diagnosis accuracy. Characteristic evaluation just provides an effective solution for the above problems. On the basis of certain evaluation criteria, the sensitivity of each characteristic parameter is learned, and the original characteristic set is filtered or weighted according to the learning results, which thus plays the purpose of improving the characteristic clustering and classification performance. Most of the widely used feature weighted methods use the weight coefficient (weight coefficient) as the core content [22] and screen the features and weighting of high Vite solicitation based on a single sensitivity evaluation model. At present, the feature evaluation model uses more distance, consistency, information, correlation measures, etc. [26] Common distance measures are pasteurized distance, Euclidean distance, and Marchhedean distance; information measure mainly includes mutual information and information gain; consistency measure is the main indicator; correlation measure includes Pearson (Pearson) correlation coefficient [27], minimum square regression error, probability error, Fisher score, Laplacian score, linear classification and analysis, and high calculation efficiency. The above single measurement feature evaluation methods can screen deredundancy features from a specific perspective, but their limitations are strong, which cannot be comprehensive screening features and fully reflect the protection information. The existing feature-weighted methods are realizing the learning and weighted processing of feature sensitivity based on a feature evaluation model of a single measure, ignoring the complementary effect of the multimeasure evaluation model and the composite gain effect on high-dimensional feature screening. Therefore, the experimental mixed measure feature evaluation model is composed of a random combination of $n$ typical feature evaluation models in each single measure index benchmark, and the specific implementation process is shown in Figure 5. Different measure combination mixed strategies form $Q=C_{n}^{2}+C_{n}^{3}+\cdots C_{n}^{n}$ and then select the optimal measure combination strategy with the maximum variation coefficient. Secondly, the multimeasure optimal mixed 


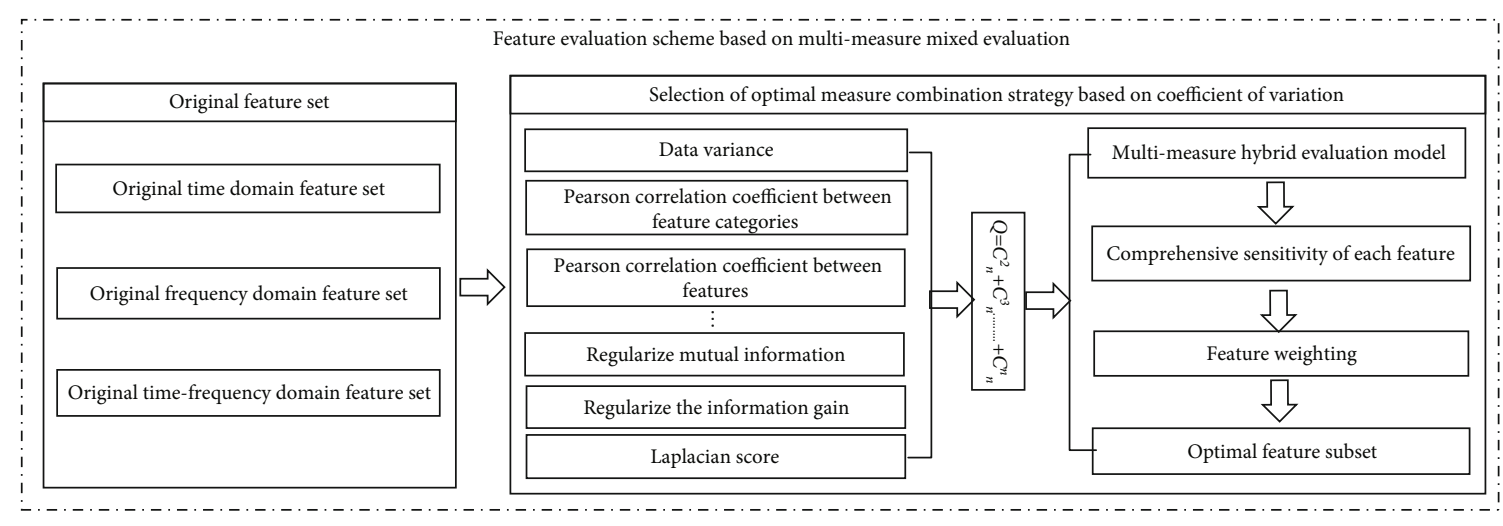

FIgURE 5: An evaluation model based on multimeasure mixing.

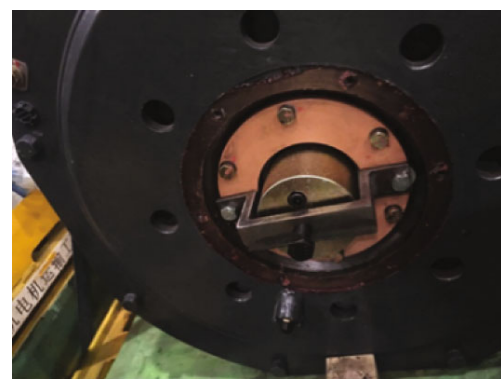

(a) The screw on the nondrive end is top dead

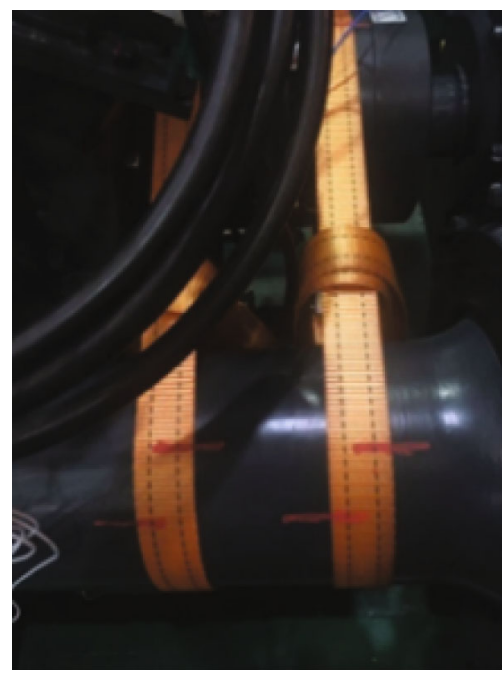

(b) The drive end is fixed by the strap

Figure 6: Protection status 1 setting.

evaluation model is calculated as the combined feature weight and the original eigenvalue. The detailed flow is shown in Figure 5.

Ten common feature evaluation models are selected as alternative subevaluation models for the mixed measure strategy model. It includes information measurement, correlation measure, and distance measure feature evaluation model. Due to the large number of mixed measure models with random free combination of different single measures, a large operational workload and too cumbersome mixed measurement strategy will produce a preferred comparison analysis process. Considering that the sensitivity of each single measure to the high-Witt solicitation varies, different feature subset dimensions remain when feature-weighted and filtered, cases where a measure removes only less nonsensitive features. The feature subsets are still too redundant, and it may even be difficult to select more sensitive features to form feature subsets for classification. Thus, initial screening and filtering of the submodel constituting the mixed measure model are required. A single measurement feature evaluation model with good sensitivity discrimination is retained. Avoid the difficulty of learning the poor compre- hensive performance of a large number of mixed measure combination strategy sensitivity, resulting in the optimization process. Therefore, a preliminary selection of a single measure feature evaluation model before a mixed combination of a single measure feature evaluation model is done, and several single measure feature evaluation models with outstanding sensitivity are selected for the subsequent random mixing strategy and then the principle of the largest discrete coefficient of the mixed measure sensitivity and the largest cliff to select the best measure combination strategy.

Starting from ten common single measure feature evaluation models, the sensitivity score of time domain, frequency domain, and time-frequency domain of three different protective states of bogie is obtained of ten single measure models. It is found that different measures have different sensitivity to the feature set, some single measures are very sensitive to the feature set, and it is almost impossible to get a reduced subset of good features. Some single measures have good sensitivity learning effect to the feature set, high sensitivity features are more prominent, and nonsensitivity features are weakened, making the feature sensitivity differentiation more obvious. This section preliminarily selects 


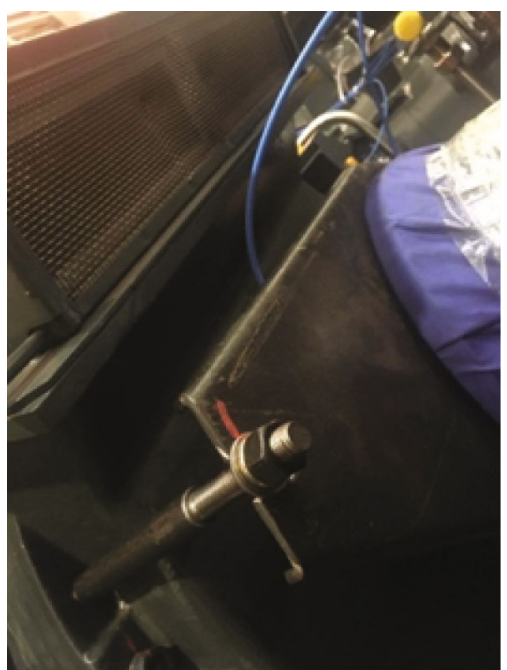

(a) Drive end decoupling pressure plate plus tie rod protection

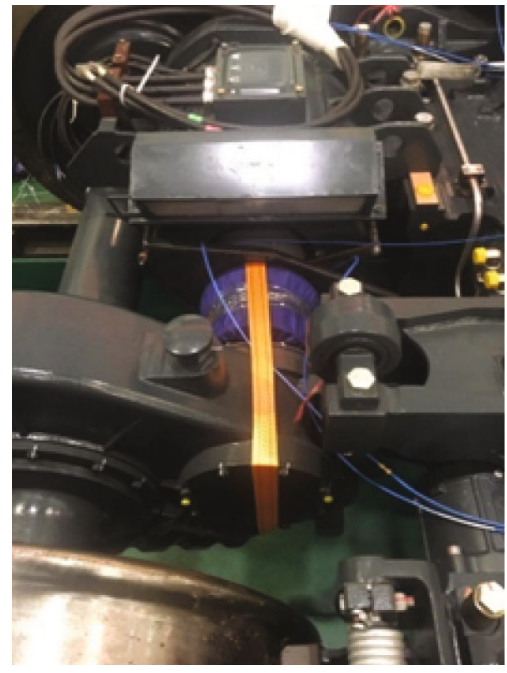

(b) Drive end decoupling coupling fixing belt

Figure 7: Protection status 2 setting.

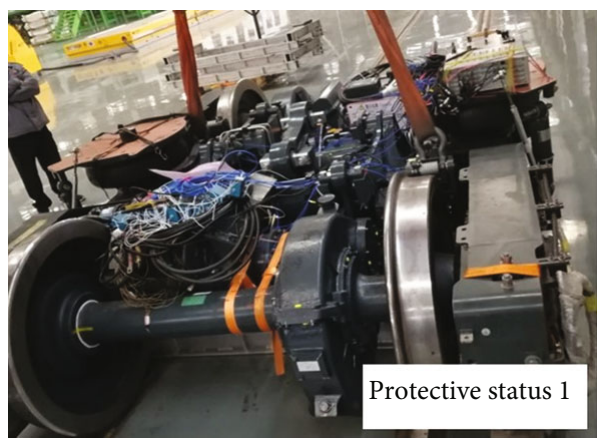

(a)

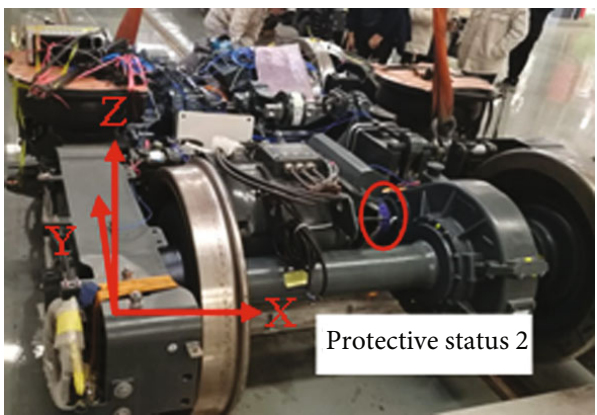

(b)

FiguRE 8: Overall layout of the protection scheme.

TABLE 1: Summary of protection status of motor bearings.

\begin{tabular}{lcccc}
\hline Protective status & Drive end & Nondrive end & Fixed binding of transmission end & Back and forth \\
\hline 1 & Undecoupled & Screw top is dead & Longitudinal with the wheel axis & Go/return \\
2 & Decoupled & Screw top is dead & Unfastened & Go \\
3 & Undecoupled & The screw is not dead & Return \\
\hline
\end{tabular}

ten single measure evaluation models and is selected as the submodel of the subsequent mixed measure feature evaluation model. Because the dimension of each measure is inconsistent, but the dimension of the feature on each single measure is comparable, a uniformly defined threshold $\sigma^{i}=$ $\left(\sum_{l}^{j} \lambda_{j}^{i}\right) / j, \lambda_{j}^{i}$ is the type $j$ feature sensitivity score of a single measure which is set for each single measure. If the sensitivity score of more than 10 characteristics of a single measure is greater than the mean value $\sigma^{i}$, the sensitivity score learning effect of the single measure is poor, and if the single measure is abandoned, the submodel for the subsequent evaluation model of mixed measurement characteristics is retained. Secondly, construct a mixed measure feature sensitivity learning model with cumulative effect composed of individual measures, whose mathematical expression is as follows:

$$
w_{j}^{H}=\frac{w_{j}^{1} \times w_{j}^{2} \times \cdots \times w_{j}^{p}}{w_{j}^{p+1} \times w_{j}^{p+2} \times \cdots \times w_{j}^{n}} .
$$

In the formula, $w_{j}^{H}$ represents the comprehensive measure sensitivity value of the $j$ feature of the sample feature set in the $H$ hybrid strategy; $w_{j}^{1}, \mathrm{w}_{j}^{2}, \cdots, w_{j}^{n}$, respectively, represents the $1,2,3, \cdots, n$ kind single measure sensitivity value result of a sensitivity learned $j$ feature in the feature set of a single measure feature evaluation model of $w_{j}^{1} \sim$ $w_{j}^{p}$ which is a sensitivity learning value that is positively 


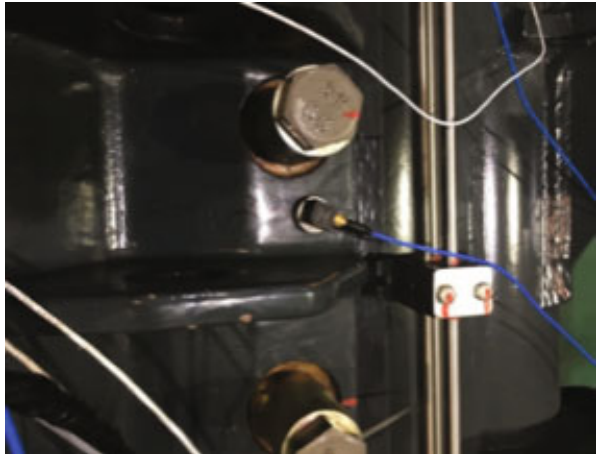

(a) Sensor 1

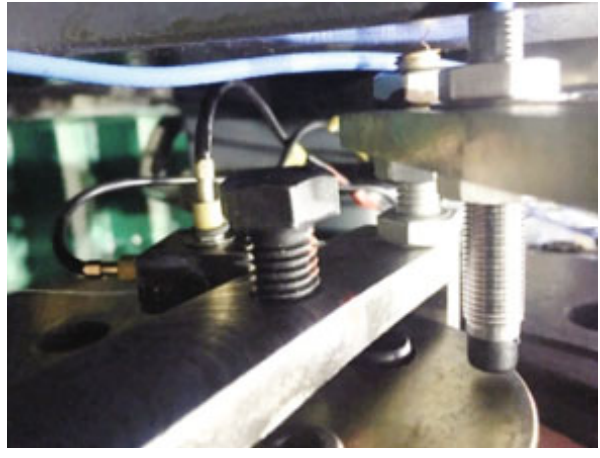

(c) Sensor 3

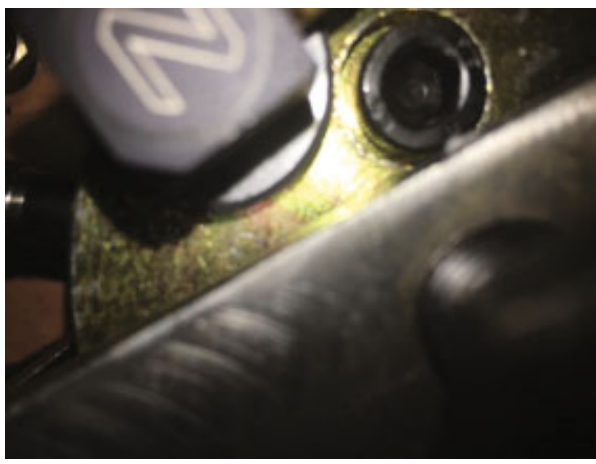

(e) Sensor 5

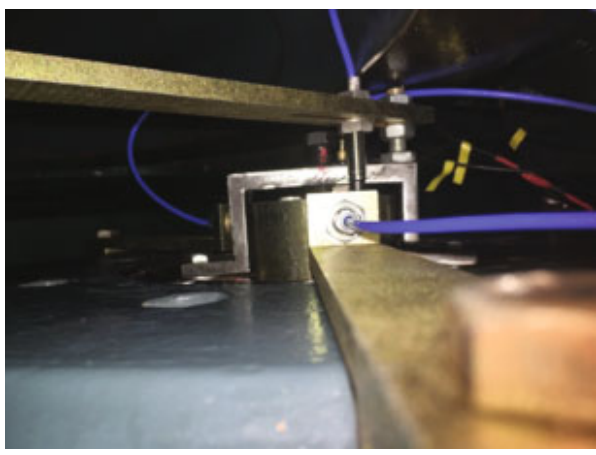

(g) Sensor 7

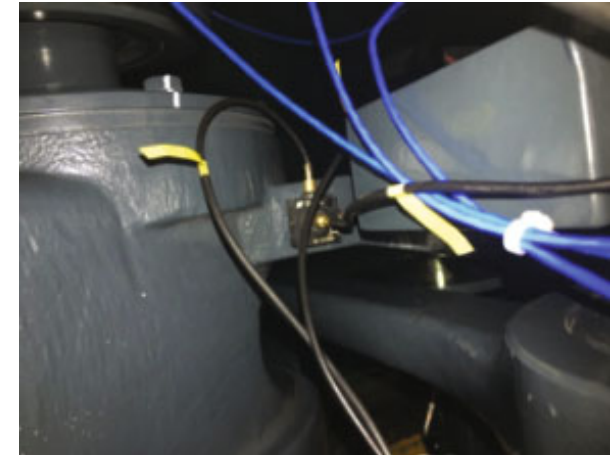

(b) Sensor 2

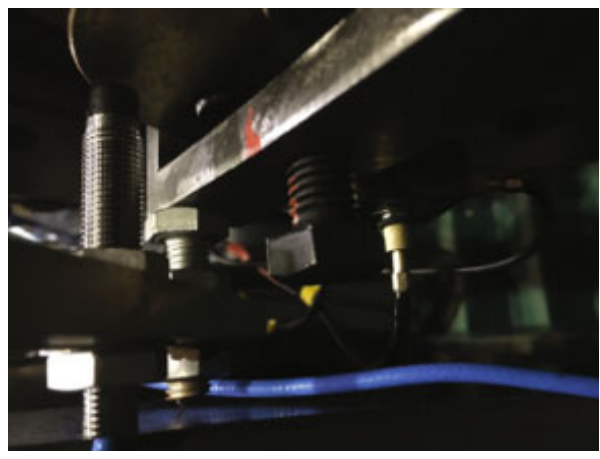

(d) Sensor 4

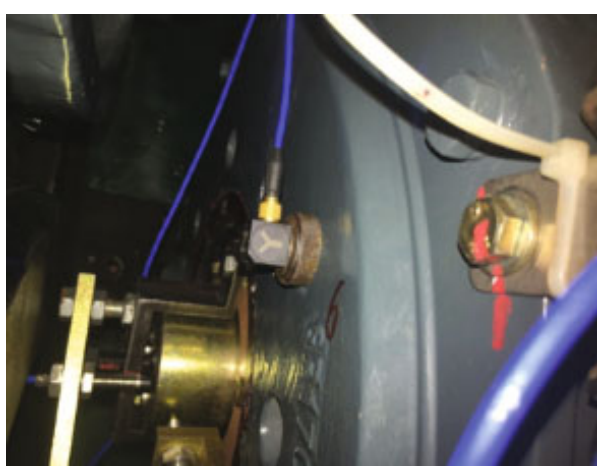

(f) Sensor 6

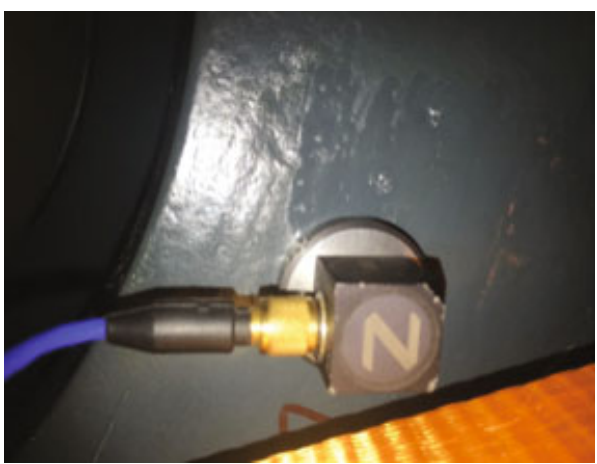

(h) Sensor 8

FIGURE 9: Sensor measuring point layout.

related with the characteristic sensitivity of the characteristic sensitivity of a single measurement evaluation model. $w_{j}^{p} \sim w_{j}^{n}$ is the sensitivity learning value of the first feature sensitivity score of the single measure evaluation model and the characteristic sensitivity of the single measurement model. Among them is $1 \leq p \leq n ; 1 \leq n \leq 4$. In order to 


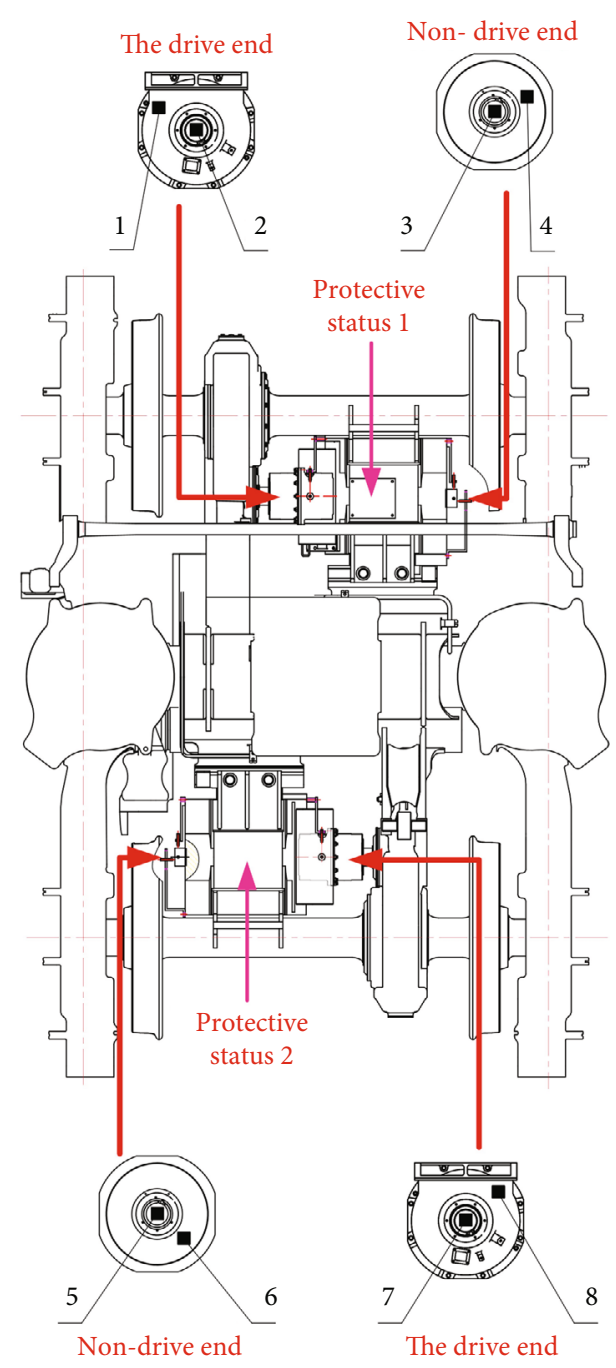

FIGURE 10: Schematic layout of sensor points on the bogie traction motor bearings.

avoid the diversity of sensitivity learning results of different single measure feature evaluation models, affecting the sensitivity learning of mixed measure evaluation models, the sensitivity score value $w_{j}^{i}$ of each single measure model should be normalized. The formula for the normalization of the comprehensive measure sensitivity score $w_{j}^{i}$ of the type $j$ feature in the type $i$ hybrid model is as follows:

$$
\tilde{w}_{j}^{i}=1+9\left(\frac{w_{j}^{i}-\min \left(\mathrm{w}^{i}\right)}{\max \left(w^{i}\right)-\min \left(w^{i}\right)}\right) .
$$

After product mixing, the comprehensive measure sensitivity learning results $w_{j}^{i}$ are obtained. The significance is to compare and evaluate the comprehensive measure sensitivity scores $w_{j}^{i}$ obtained from the product effects of different scales under the same scale coefficient, which also makes the sensitivity of high-sensitivity features more prominent and weakens the effect on classification and

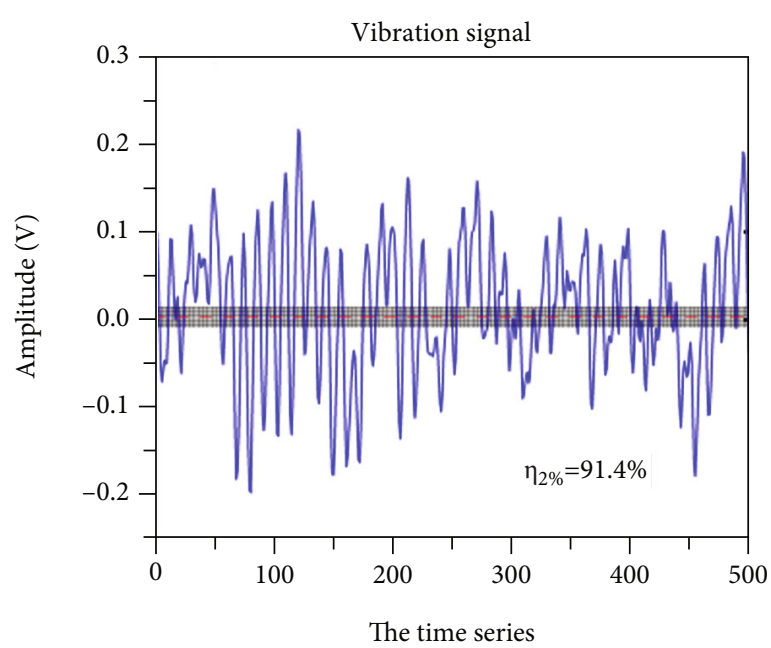

Figure 11: Vibration signal of traction motor bearing.

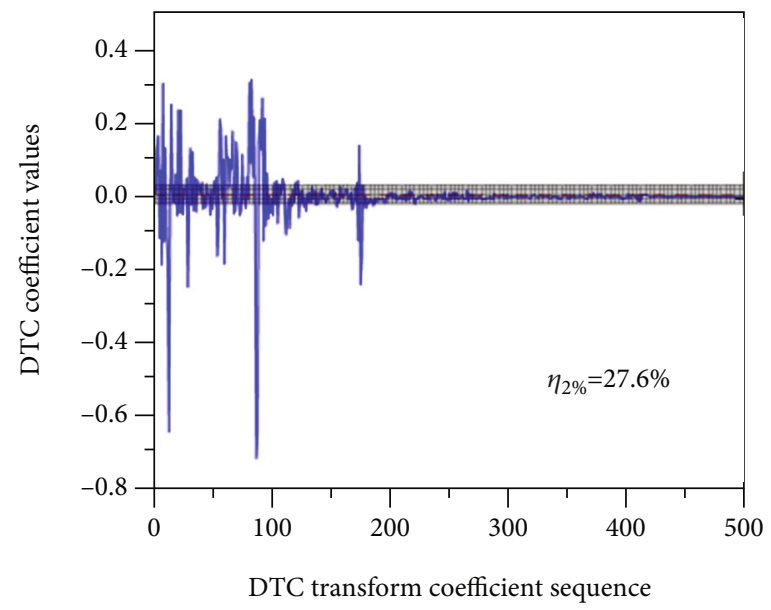

FiguRE 12: DCT sparse transformation.

clustering in the nonsensitivity features. Thus, the clustering effect of the optimal feature subset is improved.

In order to evaluate the multimeasure mixed evaluation model after a single measure feature evaluation model, the multimeasure evaluation model for the multimeasure evaluation model with the maximum discrete coefficient and the maximum cliff and extract the sensitive feature subset of the best combination strategy for subsequent clustering and evaluation. The core idea is a dimensionless parameter index that reflects the degree of data discretization according to the discretization coefficient and cliff of the time series. The specific criterion of $\Psi_{m}=\sqrt{V_{m} \times K_{m}}$ is that the greater the cliff value $K_{m}$ and the greater the discrete coefficient $V_{m}$ of the mixed characteristic sensitivity score sequence, the higher the discrete degree of the characteristic data sequence and the greater the $\Psi_{m}$ value. The reaction performance in the feature concentration is strengthening the feature sensitivity learning effect with high sensitivity and weakening the learning effect of nonsensitivity features. Make the high-sensitivity features stand out in the mixed measure strategy. Thus, it can be considered that the higher the cliff value of the eigenvalue and the greater the discrete 


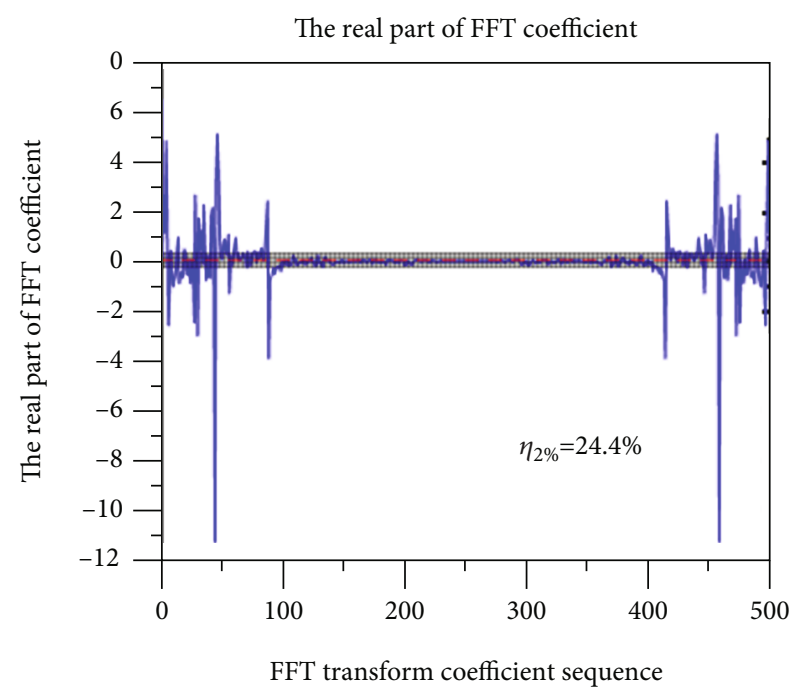

(a) Discrete Fourier sparse transform (real part)

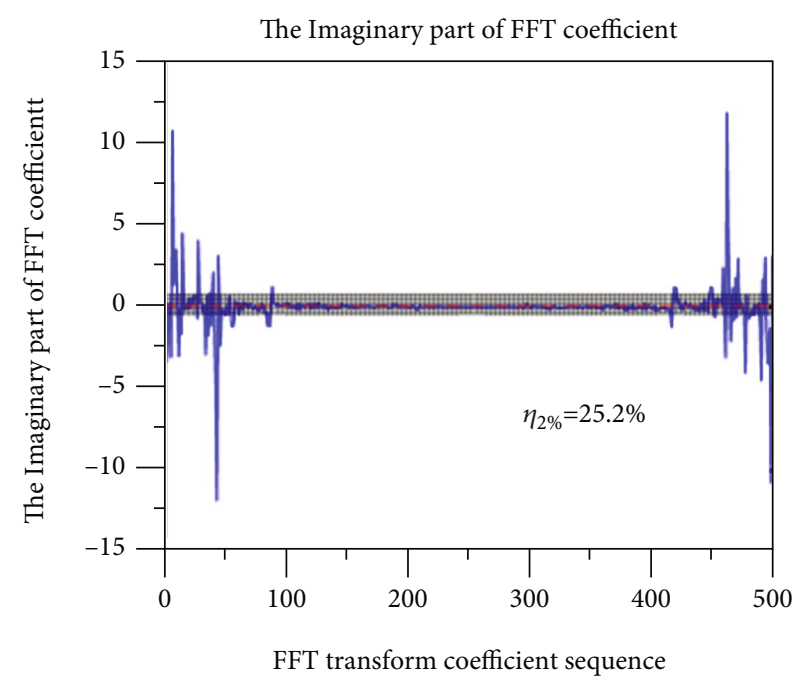

(b) Discrete Fourier sparse transform (imaginary part)

FIgURE 13: DFT sparse transformation.

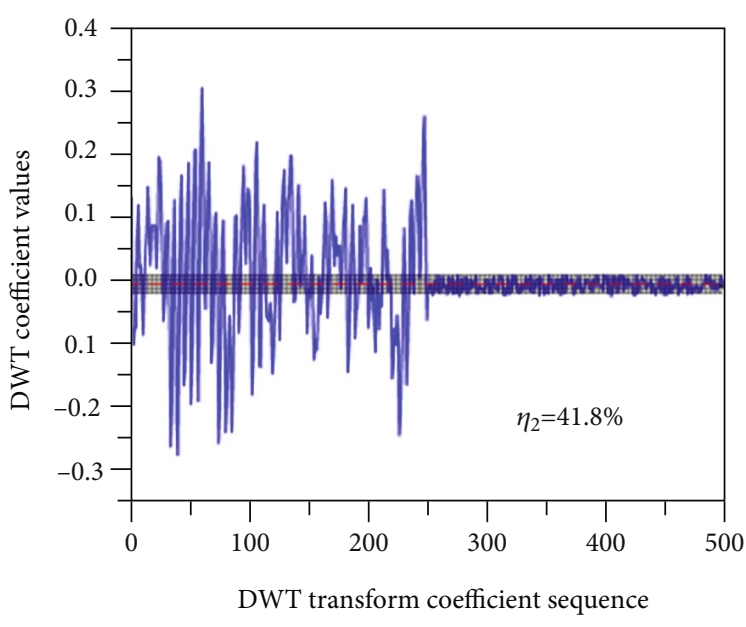

(a) Wavelength $=8$

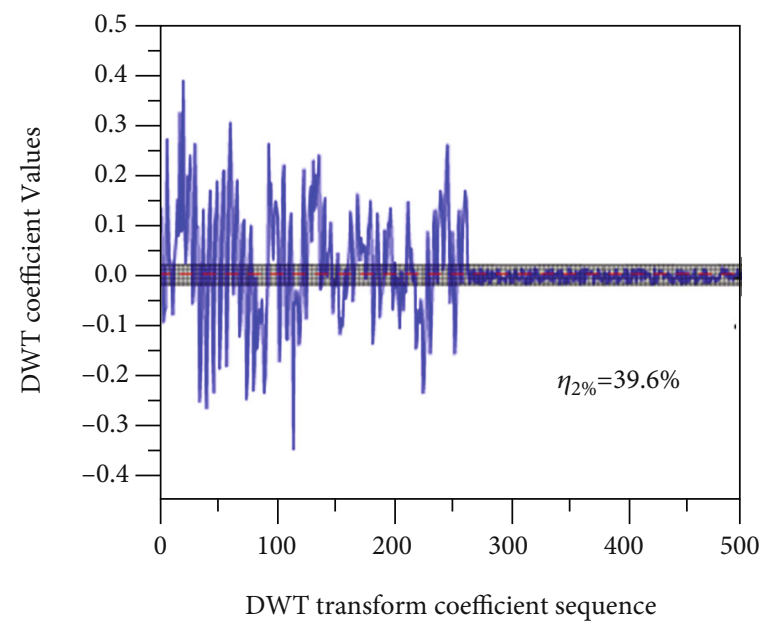

(b) Wavelength $=12$

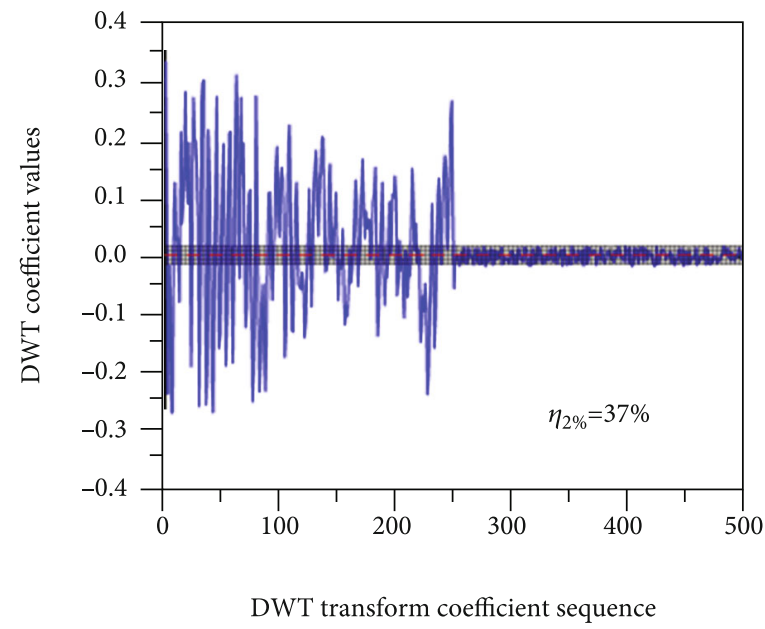

(c) Wavelength $=16$

FIGURE 14: Discrete wavelet transform. 
coefficient of the mixed characteristic sensitivity score value, the hybrid measure is best effective in sensitivity learning, and the more streamlined the subset of eigenvalue used for clustering classification. The formula for calculating the discrete coefficient of the comprehensive feature sensitivity score sequence defining $V_{m}$ as the $m$ kind mixed measure feature evaluation model is as follows:

$$
V_{m}=\frac{S_{m}}{\bar{w}^{H-m}}=\frac{\sqrt{(1 /(N-1)) \sum_{j}^{N}\left(w_{j}^{H-m}-\bar{w}^{H-m}\right)^{2}}}{\bar{w}^{H-m}} .
$$

In the formula, $S_{m}$ is the standard difference in the sequence of mixed feature sensitivity scores of type and $\bar{w}^{H-m}$ is the mean of the mixed feature sensitivity score series of the $m$ kind mixed measure feature evaluation model: $\bar{w}^{H-m}=\left(\sum_{j=1}^{N}\left(w_{j}^{H-m}-\bar{w}^{H-m}\right)\right) / N, N$ is the total number of features in the feature set, and $w_{j}^{H-m}$ is the comprehensive sensitivity score of the $j$ feature of different protective state features in the $m$ kind mixed measure feature evaluation model. The formula defining the cliff value $K_{m}$ of the $m$ kind mixed measurement feature evaluation model is as follows:

$$
K_{m}=\frac{E\left(x_{m}-\bar{x}_{m}\right)^{4}}{\sigma_{m}^{4}} .
$$

In the formula, $x_{m}, \bar{x}_{m}$, and $\sigma_{m}$ are the data series, mean, and standard difference of the mixed measure feature evaluation model of type $m$, respectively.

The specific criterion of $\Psi_{m}=\sqrt{V_{m} \times K_{m}}$ is based on the greater the cliff value $K_{m}$ and the greater the discrete coefficient $V_{m}$ of the mixed characteristic sensitivity score sequence, the higher the characteristic data sequence and the greater the $\Psi_{m}$ value. The reaction performance in the feature concentration is strengthening the feature sensitivity learning effect with high sensitivity and weakening the learning effect of nonsensitivity features. Make the high sensitivity features stand out in the mixed measure strategy. Thus, it can be considered that the higher the cliff value of the eigenvalue and the greater the discrete coefficient of the mixed characteristic sensitivity score value, the hybrid measure is best effective in sensitivity learning and the more streamlined the subset of eigenvalue used for clustering classification.

The traditional feature evaluation model with a single measure usually considers only from a single angle when extracting the optimal feature subset, which makes it difficult to evaluate the vibration signals in the whole time domain under different protection conditions. In order to better evaluate the protection effect of traction motor bearings under different protection conditions during transportation, this chapter proposes a multimeasure mixed evaluation model, which can extract multicategory and multimeasure characteristic parameters as feature sets. The novelty lies in that based on the feature index, sensitivity learning is carried out for the features in the multianalysis domain, a multimeasure mixed evaluation model is established based on the sin-

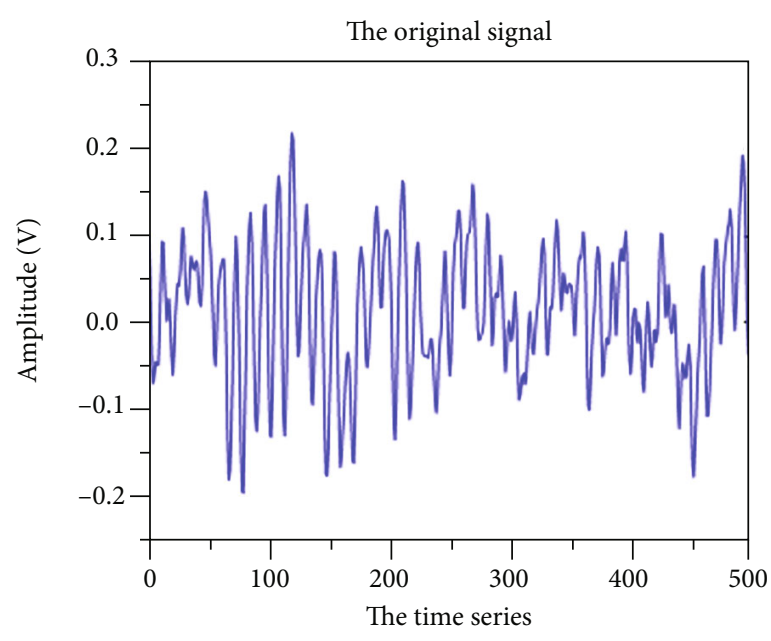

FIgURE 15: Original vibration signal.

gle measure feature evaluation feature index, and the optimal feature subset that is more conducive to evaluating the effects of different protection states is optimized by using the comprehensive feature evaluation index. Then, the unified feature index was established based on the optimal feature subset to comprehensively evaluate the protection effect under different protection conditions.

4.4. Build a Multimeasure Hybrid Evaluation Model Based on Compression Sensing. In this paper, based on the actual operating conditions of bogie traction motor bearings in urban rail vehicles, because the bearings are difficult to be effectively evaluated under different transportation protection, a multimeasure hybrid evaluation model based on compressive sensing is proposed. Firstly, vibration signals of traction motor bearing are sampled and compressed based on compressive sensing theory. Secondly, an optimal hybrid model feature evaluation framework with single measures such as distance, correlation, and information is constructed to learn feature sensitivity from the original feature set composed of time-frequency, frequency-domain, and frequency-domain feature parameters. At the same time, to design comprehensive features based on sensitivity score sequence variation coefficient of the multimeasure portfolio strategy optimization method and mixed with the optimal combination strategy measure corresponding comprehensive evaluation model to the original fault feature set sensitivity study and then the optimal comprehensive score of each feature sensitivity for each feature weights, build a new weighted feature set. Finally, the proposed feature sensitivity learning method is applied to the transportation of bogie traction motor bearings of Ningbo Line 3 urban rail vehicles, so as to verify the feasibility and technical advantages of the proposed new method. This method provides a technical basis for the difficulties existing in the traction motor bearings of transit sentence frame in the field of rail transit and provides a new idea for the data collection and reduction of vibration signals of bearings in the process of transportation, as well as reasonable transportation protection. 


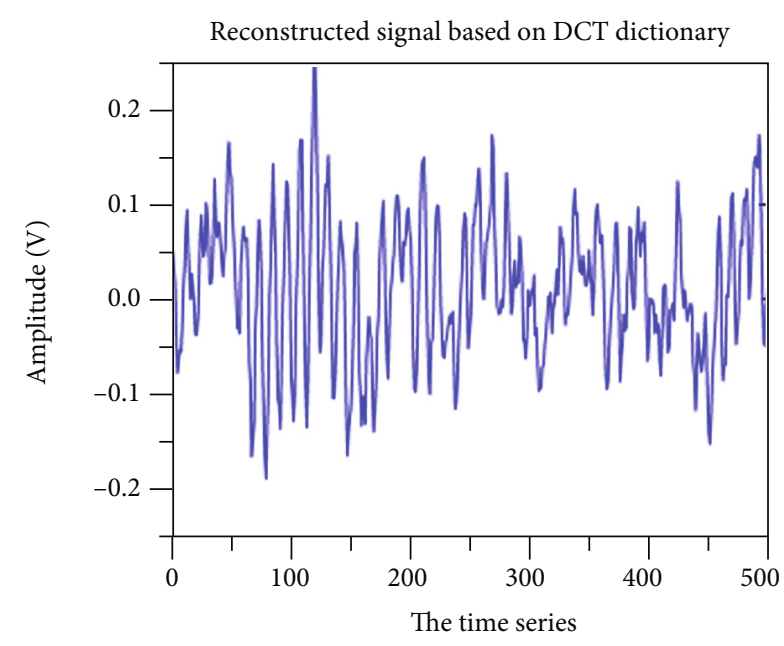

(a) Reconstruction signal based on DCT representation

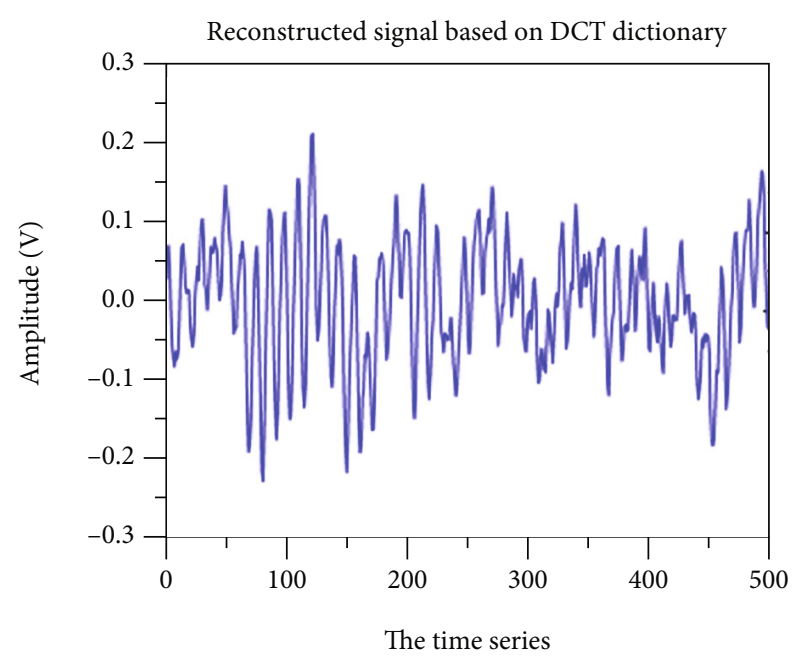

(b) Reconstruction signal based on DFT representation

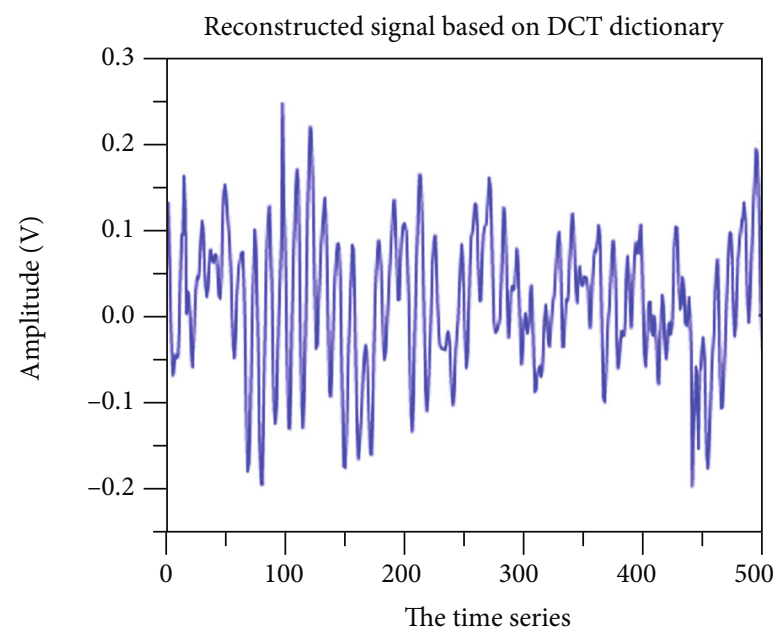

(c) Reconstruction signal based on DWT representation

Figure 16: Comparison of reconstructed signals.

\section{Experimental Analysis}

\subsection{Experimental Measurement}

5.1.1. Three Protection States of Traction Motor Bearings. The test object is the power bogie of Ningbo Line 3. The bogie wheel pair is supported and fixed with iron shoes. In the transportation process, the bogie two guided motors adopt different protection methods to test the vibration acceleration of bogie and motor bearings in Zhuzhou to Ningbo section. In the process of road transportation, motor bearings are protected as shown in Figure 6. Protection status 1 and protection status 2 are used for the two motors, respectively, during the departure, and protection status 1 remains unchanged while protection status 2 is changed to protection status 3 during the return, so as to study the protective effects of the three protection status on traction motor bearings.

Figure 6 shows the defense mode in defense state 1, Figure 7 shows the defense mode in defense state 2, and Figure 8 shows the overall layout in defense state. In

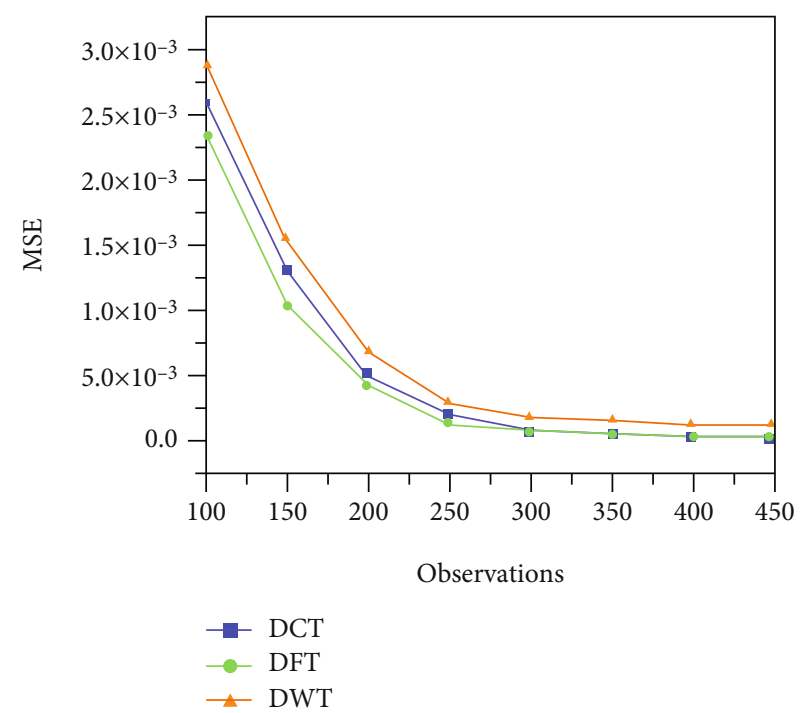

FIgURE 17: Mean square errors of reconstruction of different sparse representation methods. 


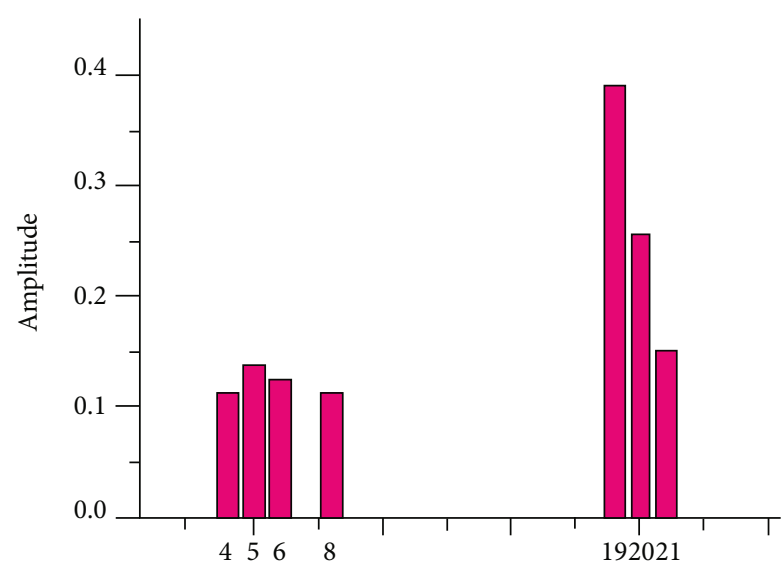

Characteristics of the serial number

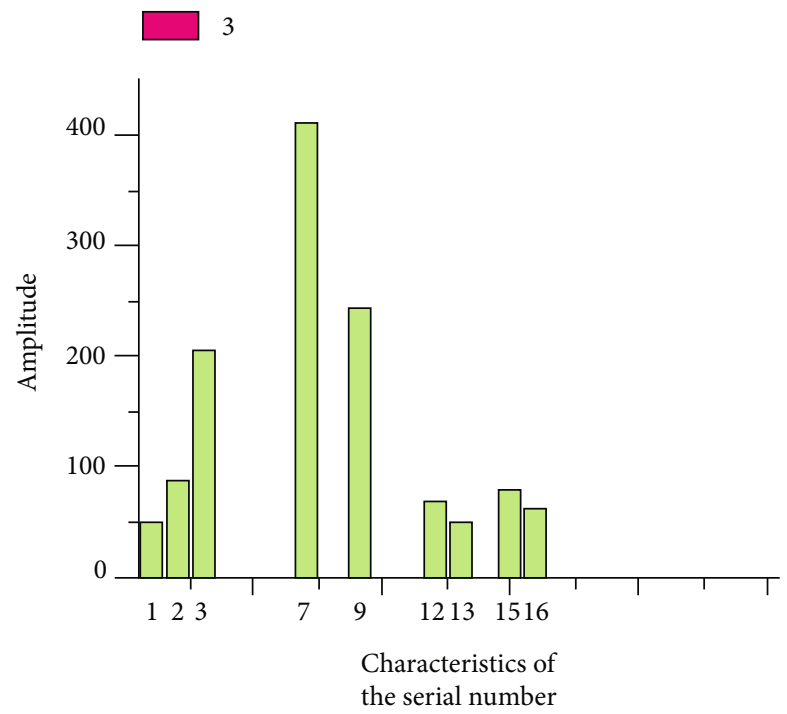
7

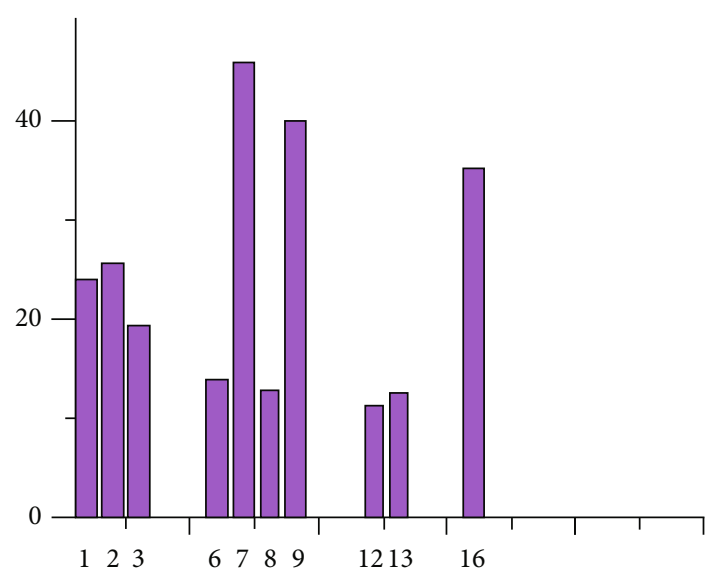

Characteristics of the serial number

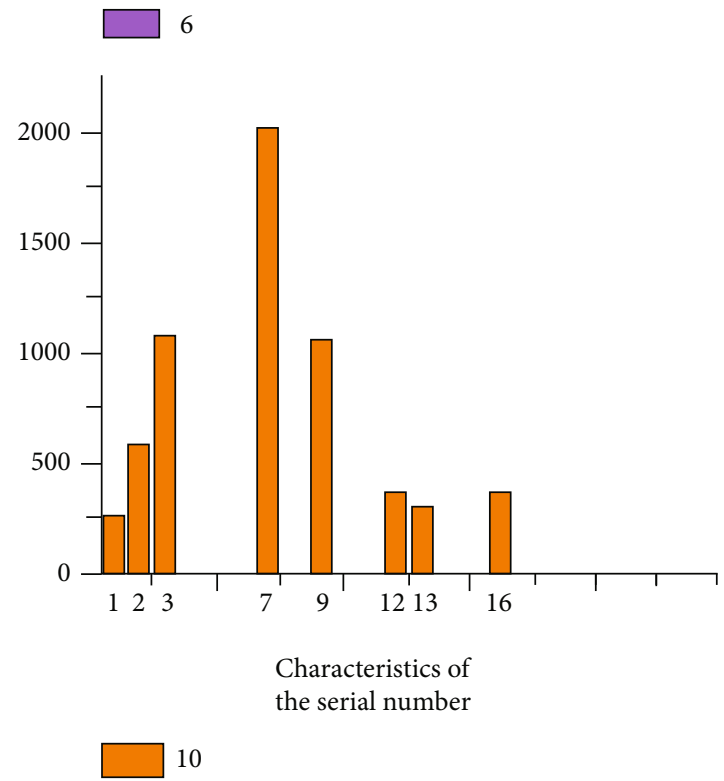

FIGURE 18: Preferred sensitivity score of a single measure sensitivity feature.

addition, the coordinate directions of the three-way acceleration sensor are defined. Since the vertical direction along the $Z$ direction is most affected by road turbulence, the analysis of vertical vibration acceleration signal is mainly carried out. Table 1 shows three settings of the same protection state.

5.1.2. Measuring Point Arrangement of Traction Motor Bearing. A total of 8 acceleration sensors are arranged on the bogie, and the acceleration sensors at each measuring point are fixed by glue and magnetic suction seat. According to the designed sensor point position and sensor type, the sensor is arranged on the bogie traction motor. According to the position of the traction motor bearing in the bogie, in order to better collect the bearing vibration signal in the process of traction motor in the road, the arrangement of vibration sensor measuring points is shown in Figure 9. Figures 10(a)-10(h) correspond to sensors numbers 1-8.
5.2. Bearing Data Compression Perception. After the experimental test, the experimental data was up to $254 \mathrm{~GB}$ and was difficult for subsequent data analysis. This paper proposes a method to solve the problems of data mass and redundant data. Firstly, the vibration signal of a transport bearing is randomly selected to conduct compression sensing sparse representation analysis, and the DCT, DFT, and DWT transformation of the experimental signal are performed. In Figure 11, the sparse ratio of the original vibration signal is $91.4 \%$. Figure 12 intuitively shows that the sparse ratio is $27.6 \%$ after the DCT transformation, and the sparsity of the vibration signal after the DCT transformation is higher than that of the original signal. Figure 13 is the result of the vibration signal after the DFT transformation, and the sparse ratio of the DFT coefficient is $24.4 \%$ and $25.2 \%$, respectively, which is more prominent compared with the DCT transformation. Figure 14 shows that the sparsity of the vibration signal is well improved compared with the original signal. The sparse score of Daubechies wavelet 
TABLE 2: Results of various evaluation parameters of characteristic sensitivity score of multimeasure mixed model.

\begin{tabular}{|c|c|c|c|c|c|c|}
\hline $\begin{array}{l}\text { Hybrid policy } \\
\text { Group-up }\end{array}$ & $\begin{array}{l}\text { A single } \\
\text { type of } \\
\text { measure } \\
\text { included }\end{array}$ & $\begin{array}{c}\text { Sensitivity learning } \\
\text { score sequence } \\
\text { standard difference } \\
S_{m} \\
\end{array}$ & $\begin{array}{l}\text { Sensitivity scores } \\
\text { learn the score } \\
\text { sequence } \\
\text { mean } \bar{w}^{H-m} \\
\end{array}$ & $\begin{array}{c}\text { Sensitivity scores learn } \\
\text { the score sequence } \\
\text { discrete coefficients } \\
V_{m}\end{array}$ & $\begin{array}{l}\text { Sensitivity scores } \\
\text { learn the score } \\
\text { sequence cliff } \\
\text { values } K_{m} \\
\end{array}$ & $\begin{array}{l}\text { Comprehensive } \\
\text { evaluation index of } \\
\text { sensitivity score } \\
\text { sequence } \Psi_{m}\end{array}$ \\
\hline$\omega^{H-1}$ & $3 \#, 6 \#$ & 2.5450 & 3.5357 & 0.7198 & 3.2073 & 1.5194 \\
\hline$\omega^{H-2}$ & $3 \#, 7 \#$ & 2.1382 & 2.1456 & 0.9966 & 8.8875 & 2.9761 \\
\hline$\omega^{H-3}$ & $3 \#, 10 \#$ & 2.1179 & 2.2097 & 0.9585 & 8.7751 & 2.9001 \\
\hline$\omega^{H-4}$ & $6 \#, 7 \#$ & 1.9732 & 1.7685 & 1.1158 & 13.7512 & 3.9170 \\
\hline$\omega^{H-5}$ & $6 \#, 10 \#$ & 1.9489 & 1.7830 & 1.0931 & 14.1195 & 3.9286 \\
\hline$\omega^{H-6}$ & $7 \#, 10 \#$ & 1.8865 & 1.6264 & 1.1599 & 16.9705 & 4.4367 \\
\hline$\omega^{H-7}$ & $3 \#, 6 \#, 7 \#$ & 1.9730 & 1.7800 & 1.1085 & 13.6794 & 3.8940 \\
\hline$\omega^{H-8}$ & $3 \#, 6 \#, 10 \#$ & 1.9491 & 1.7938 & 1.0866 & 14.0398 & 3.9058 \\
\hline$\omega^{H-9}$ & $3 \#, 7 \#, 10 \#$ & 1.8903 & 1.6315 & 1.1587 & 16.7992 & 4.4119 \\
\hline$\omega^{H-10}$ & $6 \#, 7 \#, 10 \#$ & 1.8392 & 1.5276 & 1.2040 & 19.6013 & 4.8579 \\
\hline$\omega^{H-11}$ & $\begin{array}{c}3 \#, 6 \#, 7 \#, \\
10 \#\end{array}$ & 1.8387 & 1.5254 & 1.2054 & 19.6395 & 4.8655 \\
\hline
\end{tabular}

lengths 8,12 , and 16 is $41.8 \%, 39.6 \%$, and $37 \%$, and the change of Daubechies wavelet length has little impact on the sparsity after the coefficient transformation.

Next, consider the influence of different sparse dictionary transformations on the compression effect of the bearing vibration signal, by setting three different sparse transformation dictionaries (DCT, DFT, and DWT), in which the Daubechies wavelet length of DWT is set to 16 , selecting Gaussian random observation matrix and GOMP reconstruction algorithm. Using these three different sparse dictionaries to perform data compression measurement and reconstruct the experimental signal, we obtain the parameter indicators of the signal reconstruction performance of three different sparse dictionaries. Comparing Figures 15-17 shows that the signal reconstruction performance index of the base DFT is superior to that of DCT and DWT; in three different sparse transformation methods, DFT has better sparse performance and performance in data compression.

\subsection{Quantitative Characterization of the Bearing Protection} Effect Based on the Unified Characteristic Index. The original high-Witt collection including time domain features, frequency domain features, energy features based on EMD, and Lempel-Ziv complexity features of EMD is extracted. In order to further realize the dimensionality reduction of high-Witt collection and improve the performance of cluster classification, ten sensitivity scores as described above are learned, and the 3, 6, 7, and 10 single measure feature evaluation models are selected as the submodels of mixed multimeasure feature evaluation models according to the sensitivity learning results. They are intraclass and interclass integrated distance model, Pearson correlation coefficient model, Fisher score model, and Laplacian scoring model. The best sensitivity feature subset sensitivity score distribution under the four single measurement feature evaluation indicators is shown in Figure 18. A hybrid measure feature evaluation model is constructed for the four preferred measure evaluation submodels. Since four random combinations into mixed measure models have 11 combination methods, the 11 mixed measure feature evaluation models learn the sensitivity, obtain the comprehensive sensitivity score sequence, and obtain the discrete coefficient, cliff value, and comprehensive evaluation index of the sensitivity learning results based on the comprehensive sensitivity score evaluation index $\Psi_{m}$. The results are shown in Table 2 .

It can be seen from the table above that the discrete coefficient, cliff value, and comprehensive sensitivity score evaluation indicators of the mixed measure feature evaluation model after the random combination of four single measures are best performed when the 3,6, 7, and 10 measures are fully combined. It can therefore be argued that the hybrid strategy exhibits outstanding performance in strengthening the sensitive features, weakening the nonsensitive features, and a subset of reduced optimal features. Features with large sensitivity scores are extracted as subfeatures of the subset of optimal feature subsets. Its feature number is $1,2,3,7,9$, and 16 .

To further verify that the extracted sensitivity scores perform well for the clustering effect of a subset of optimal features, principal component analysis (PCA [28]) of the best subsets of the optimal evaluation model is applied. To facilitate the application of PCA, visual analysis is performed. Figure 19 shows a visual analysis diagram of the first characteristic subset of the 3,6,7, and 10 single measure evaluation models. It can be seen that the best characteristic subset characteristics of the 3 and 6 single measures cannot be classified and clustered by data samples regardless of the serious travel overlap between the distance and different categories. The best characteristic subset corresponding to the 7 and 10 single measure evaluation models can cluster different protective state data samples effectively, but the distribution is scattered and the inner class range is large. From 


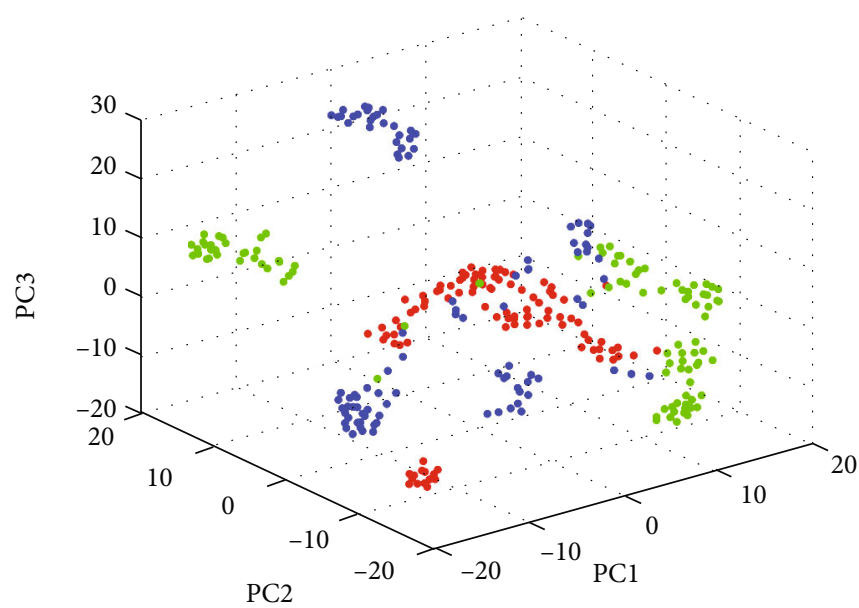

(a) The best subcluster class effect of the third single measure

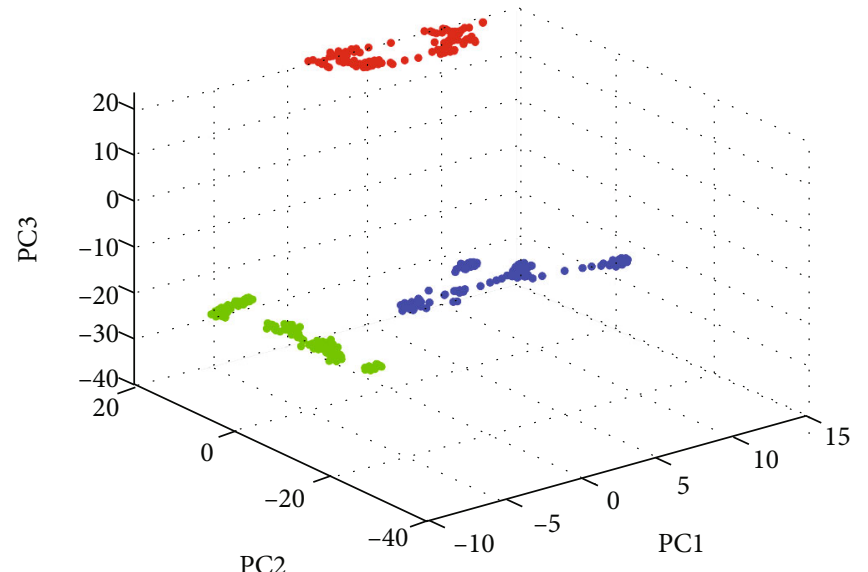

(c) The best subcluster class effect of the 7 single measure

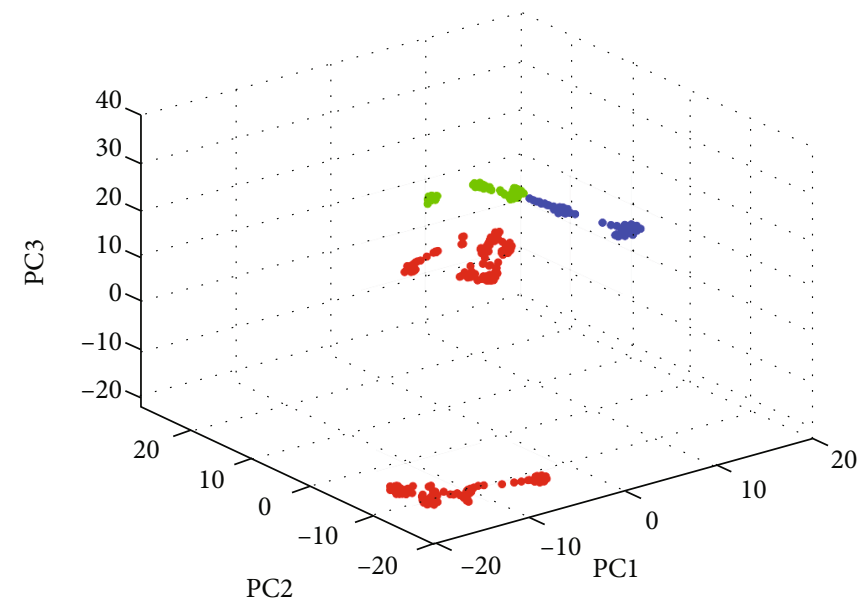

(b) The best subcluster class effect of the sixth single measure

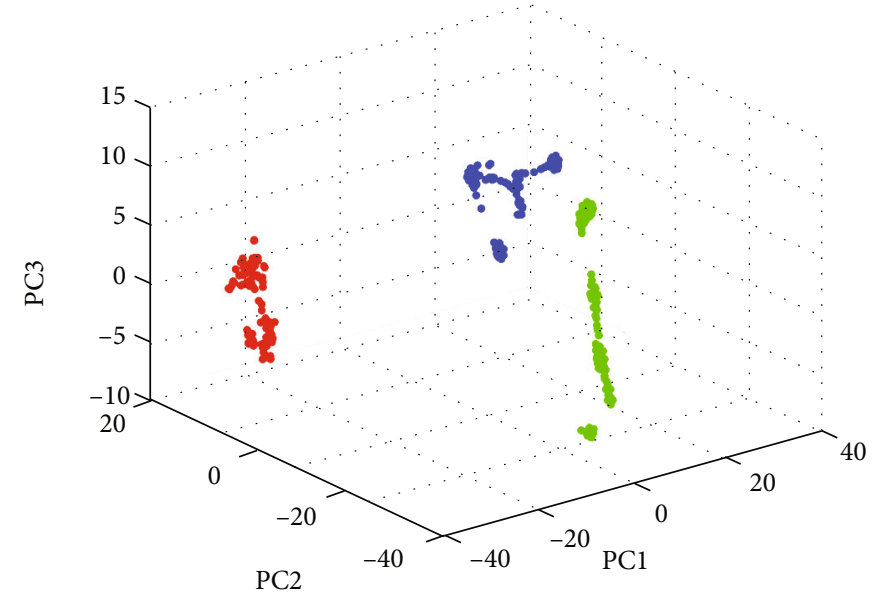

(d) Best subcluster class effect for the 10 single measure

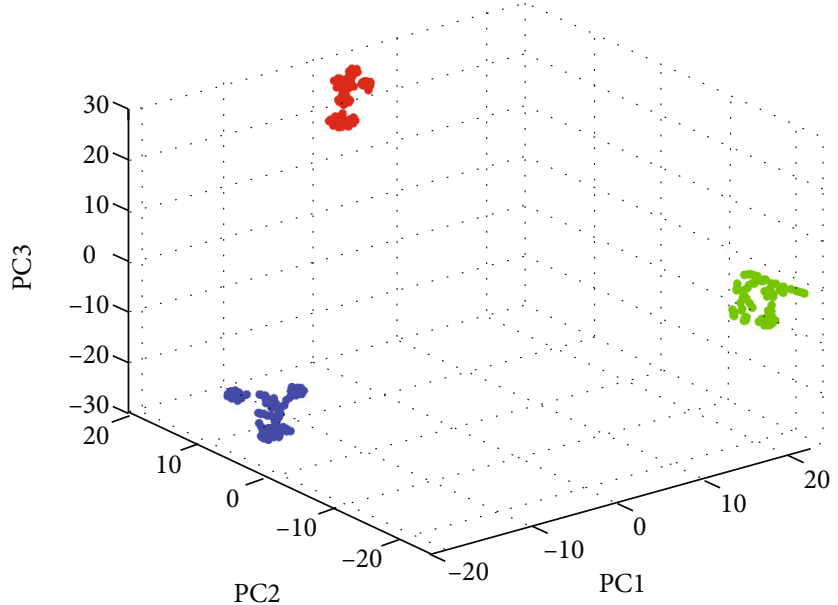

(e) Clustering effect of the optimal characteristic subset with optimal multiple measures

Figure 19: Cluster effect of the optimal subsets under different measures.

the perspective of interclass distance, the clustering effect is not prominent, and the optimal multimeasure mixed evaluation model is far better than the other four single measure subsets in classification clustering.

A Cartesian product is performed for the preferred optimal subfeature subset based on a multimeasure mixed eval- uation model. The Cartesian product calculation expressions defining the unified feature metrics are

$$
Q=\frac{v^{1} \times v^{2} \times \cdots \times v^{p}}{v^{p+1} \times v^{p+2} \times \cdots \times v^{n}}
$$



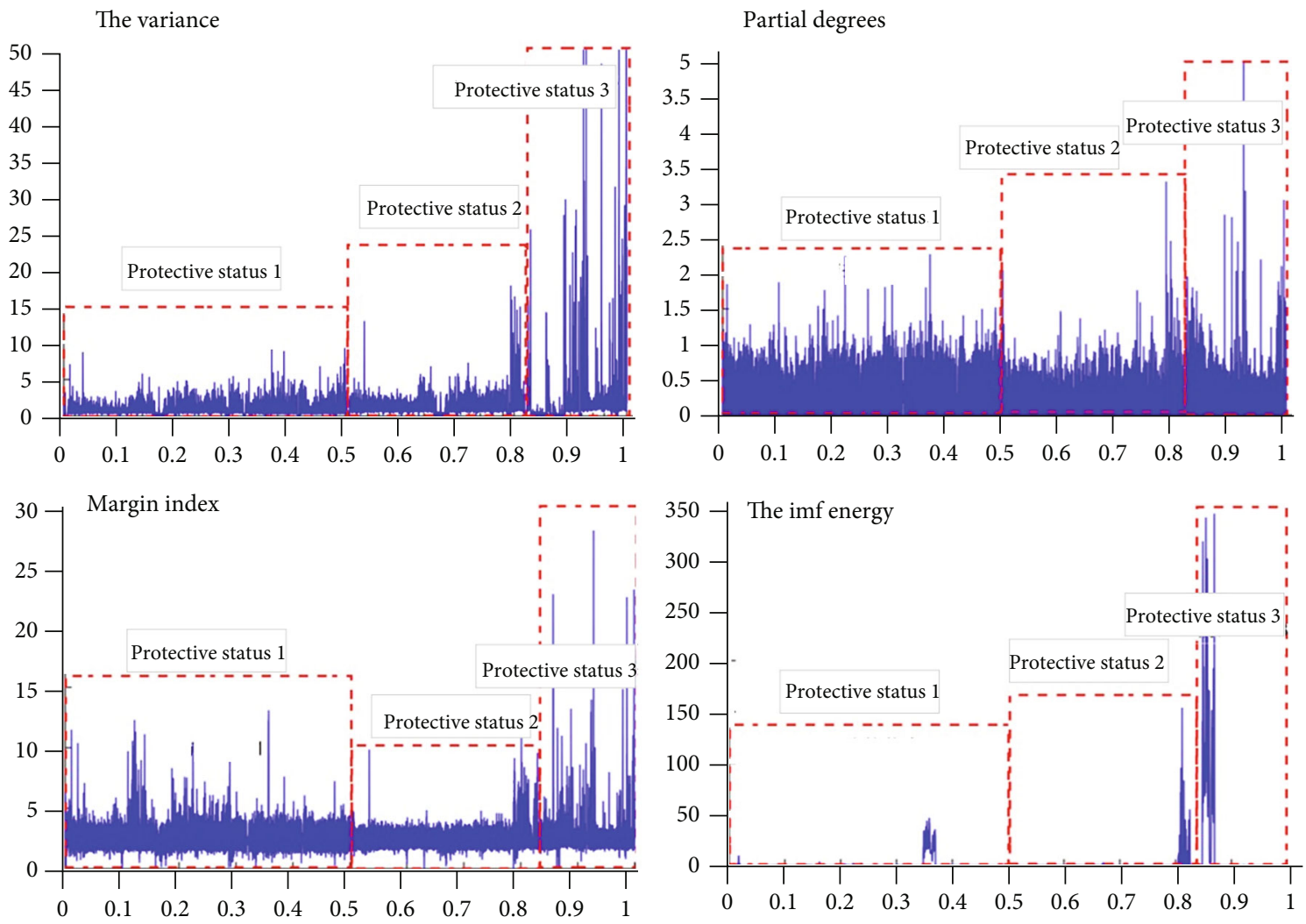

Figure 20: Part full time domain features.

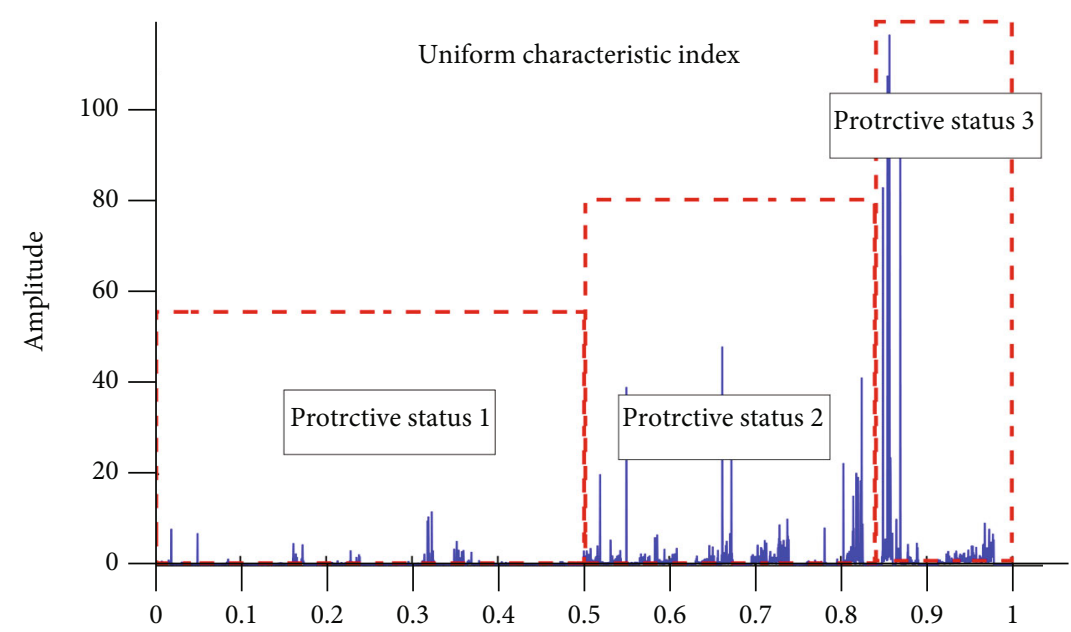

Figure 21: Comprehensive characteristic index.

In the formula, $v^{1} \sim v^{p}$ protection is negative, and $v^{p+1} \sim v^{n}$ is the positive value with the protection effect, where $Q$ is a unified feature indicator; $1 \leq p \leq n, 1 \leq n \leq 6$ . The characteristics of the characteristic subset are negatively related to the protection effect, that is, the greater the characteristics, the worse the protection effect, so the comprehensive characteristic indicators. And the larger the unified characteristic indicators, the worse the protection effect.
Figure 20 shows the distribution of different protective states in the time domain and the comprehensive evaluation from a single feature. Figure 21 is the comprehensive characteristic index of bogie bearing vibration signal after characteristic combination. The unified characteristic index based on the full time domain is the most significant in the protection state 3 (no protection) and the worst protection effect, while the unified characteristic index of protection state 1 is not significant; its peak is far less than the protection states 
2 and 3 , the best protection effect, and the protection effect of protection state 2 is secondary.

\section{Conclusion}

In this paper, the state characterization of bogie traction motor bearings under different transportation protection is studied, and the multimeasure hybrid characterization model based on compressive sensing theory is proposed, which is verified by the highway transportation example of Ningbo Line 3 urban rail vehicle bogie traction motor bearings. Experiment: when the protection state is undecoupled + the screw is not jacked (protection state 3), the bearing damage is the most serious. The second is when the protection state is decoupling + screw jacking (protection state 2). In the case that the protection state is undecoupled + binding + screw top (protection state 1 ), the bearing has almost no damage. That is, the protection effect is protection state $1>$ protection state $2>$ protection status 3 .

\section{Data Availability}

The data and the Matlab programs used to support the findings of this study are available from the corresponding author upon request.

\section{Conflicts of Interest}

The authors declared no potential conflicts of interest with respect to the research, authorship, and/or publication of this article.

\section{Authors' Contributions}

The authors' contributions are as follows. Yi Liu was responsible for guiding conceptualization and methodology, criticism, and correction. Qi Chang was responsible for conceptualization, methodology, data curation, software, validation, investigation, and original draft. Jiaxin Luo was responsible for review and editing. Lin Li was responsible for investigation, providing test guidance, and review. Junfeng Man was responsible for review and editing. Wei Fen was responsible for investigation and review. Qilin Chen was responsible for software and review. Yiping Shen was responsible for guiding conceptualization, criticism, and correction.

\section{Acknowledgments}

This research was funded by the National Key Research and Development Project (grant number 2019YFB1405400), the National Natural Science Foundation of China (grant number 51805161), and the Huxiang Youth Talent Project (grant numbers 2019RS2062 and 2020RC3049).

\section{References}

[1] D. Chunmei, H. Minggao, and L. Meiqing, "Motor bearing protection for urban rail vehicle non-rail transportation," Electric Locomotives \& Mass Transit Vehicles J, vol. 42, no. 2, pp. 62-64, 2019.
[2] E. J. Candes and T. Tao, "Near-optimal signal recovery from random projections: universal encoding strategies?," IEEE Transactions on Information Theory, vol. 52, no. 12, pp. 5406-5425, 2006.

[3] D. L. Donoho, "Compressed sensing," IEEE Transactions on Information Theory, vol. 52, no. 4, pp. 1289-1306, 2006.

[4] E. J. Candes, J. Romberg, and T. Tao, "Robust uncertainty principles: exact signal reconstruction from highly incomplete frequency information," IEEE Transactions on Information Theory, vol. 52, no. 2, pp. 489-509, 2006.

[5] Weifen, Key Technology Research for Intelligent Fault Diagnosis of Rotating Mechanical Multi-Sensor Information Fusion, Harbin University of Technology, China, 2018.

[6] D. Xiao, C. Qin, H. Yu, Y. Huang, and C. Liu, "Unsupervised deep representation learning for motor fault diagnosis by mutual information maximization," Journal of Intelligent Manufacturing, vol. 32, no. 2, pp. 377-391, 2021.

[7] J. Luo, J. Huang, and H. Li, “A case study of conditional deep convolutional generative adversarial networks in machine fault diagnosis," Journal of Intelligent Manufacturing, vol. 32, no. 2, pp. 407-425, 2021.

[8] Z.-C. Liao, X.-X. F. Bai, Y. Li, X.-C. Deng, and J. Sun, "Design, modeling, and verification of a test bench for braking simulation of 1/4 vehicle," Proceedings of the Institution of Mechanical Engineers, Part D: Journal of Automobile Engineering, vol. 234, no. 5, pp. 1425-1441, 2020.

[9] L. Qiang, Z. Shuo, and W. Yujun, "A dynamic response test rig of a full-scale rotor-journal bearing system," Proceedings of the Institution of Mechanical Engineers, vol. 233, no. 4, pp. 649659, 2019.

[10] Y. He, K. Feng, M. Hu, and J. Cui, “An MCM-enhanced compressive sensing for weak fault feature extraction of rolling element bearings under variable speeds," Shock and Vibration, vol. 2020, 21 pages, 2020.

[11] J. Yang, X. Liao, X. Yuan et al., "Compressive sensing by learning a Gaussian mixture model from measurements," IEEE Transactions on Image Processing, vol. 24, no. 1, pp. 106-119, 2015.

[12] F. Krahmer, D. Needell, and R. Ward, "Compressive sensing with redundant dictionaries and structured measurements," SIAM Journal on Mathematical Analysis, vol. 47, no. 6, pp. 4606-4629, 2015.

[13] Q. H. Dai, "Research on compressed sensing," Chinese Journal of Computers, vol. 34, no. 3, pp. 425-434, 2011.

[14] D. L. Donoho and X. Huo, "Uncertainty principles and ideal atomic decomposition," IEEE Transactions on Information Theory, vol. 47, no. 7, pp. 2845-2862, 2011.

[15] M. El Ad and A. M. Bruckstein, "A generalized uncertainty principle and sparse representation in pairs of bases," IEEE Transactions on Information Theory, vol. 48, no. 9, pp. 25582567, 2002.

[16] J. A. Tropp, "Greed is good: algorithmic results for sparse approximation," IEEE Transactions on Information Theory, vol. 50, no. 10, pp. 2231-2242, 2004.

[17] R. Baraniuk, M. Davenport, R. DeVore, and M. Wakin, "A simple proof of the restricted isometry property for random matrices," Constructive Approximation, vol. 28, no. 3, pp. 253-263, 2008.

[18] E. J. Candès, "La propriete d'isometrie restreinte et ses consequences pour le compressed sensing," Comptes Rendus Mathematique, vol. 346, no. 9-10, pp. 589-592, 2008. 
[19] L. Muyang and Y. Yuan, "Study on the rolling bearing fault signal extraction technology based on compression sensing," Journal of Dalian Jiaotong University, vol. 42, no. 3, pp. 2126, 2021.

[20] Y. Lei, Z. He, Y. Zi, and X. Chen, "New clustering algorithmbased fault diagnosis using compensation distance evaluation technique," Mechanical Systems and Signal Processing, vol. 22, no. 2, pp. 419-435, 2008.

[21] S. Tang Baoping and Y. J. Zuqiang, "Fault diagnosis based on sensitive feature selection and manifold learning reduction," Journal of Vibration and Shock, vol. 33, no. 3, pp. 70-75, 2014.

[22] J. A. Sáez, J. Derrac, J. Luengo, and F. Herrera, "Statistical computation of feature weighting schemes through data estimation for nearest neighbor classifiers," Pattern Recognition, vol. 47, no. 12, pp. 3941-3948, 2014.

[23] M. Ismail and H. Frigui, "Unsupervised clustering and feature weighting based on generalized Dirichlet mixture modeling," Information Sciences, vol. 274, pp. 35-54, 2014.

[24] T. J. Yuen and R. Ramli, "Multi-objective optimization of allwheel drive electric formula vehicle for performance and energy efficiency using evolutionary algorithms," Proceedings of the Institution of Mechanical Engineers, Part D: Journal of Automobile Engineering, vol. 234, no. 5, pp. 1472-1479, 2020.

[25] L. Shen, M. Caiwen, and L. Yan, "Review of compressedsensing reconstruction algorithms," Infrared and Laser Engineering, vol. 42, no. S1, pp. 225-232, 2013.

[26] L. Zhang Fan and T. X. Jianguang, "Bearing troubleshooting based on compression sensing and multi-step feature learning," Combined machine tool and automatic machining technology, vol. 4, pp. 108-112, 2021.

[27] J. Xueling and D. Taihang, "Extraction of failure feature for electrical life prediction of AC contactor under mechanical vibration," Instrument Journal, vol. 40, no. 3, pp. 114-125, 2019.

[28] L. Jingzhang and W. Huang Xingshu, "Based on KPCA and improved K-means," Journal of South China University of Technology (Natural Science Edition), vol. 48, no. 6, pp. 143150, 2020. 